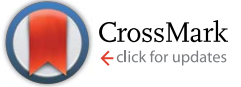

Cite this: Chem. Sci., 2015, 6, 77

Received 18th July 2014

Accepted 6th August 2014

DOI: $10.1039 / \mathrm{c} 4 \mathrm{sc} 02151 \mathrm{~g}$

www.rsc.org/chemicalscience

\section{Charge-tagged ligands: useful tools for immobilising complexes and detecting reaction species during catalysis}

\begin{abstract}
Jones Limberger, ${ }^{a}$ Bárbara C. Leal, ${ }^{a}$ Adriano L. Monteiro ${ }^{a}$ and Jairton Dupont *ab
In recent years, charge-tagged ligands (CTLs) have become valuable tools in organometallic catalysis. Insertion of an ionic side chain into the molecular skeleton of a known ligand has become a useful protocol for anchoring ligands, and consequently catalysts, in polar and ionic liquid phases. In addition, the insertion of a cationic moiety into a ligand is a powerful tool that can be used to detect reaction intermediates in organometallic catalysis through electrospray ionisation mass spectrometry (ESI-MS) experiments. The insertion of an ionic tag ensures the charge in the intermediates independently of the ESI-MS. For this reason, these ligands have been used as ionic probes in mechanistic studies for several catalytic reactions. Here, we summarise selected examples on the use of CTLs as immobilising agents in organometallic catalysis and as probes for studying mechanisms through ESI-MS.
\end{abstract}

\section{Introduction}

Undoubtedly, the choice of an appropriate solvent is vital for any organic transformation. ${ }^{1}$ The reaction media can dramatically affect the yield and selectivity of a reaction, owing to several factors including the reagents, the solubility of the products and catalysts as well as charge stabilisation. The importance of this issue is increased in homogeneous biphasic catalysis. Indeed, an incorrect solvent pairing can lead to low conversions, poor product extraction, no phase separation and catalyst lixiviation. ${ }^{2}$ Historically, one of the best strategies to avoid lixiviation is to insert organic moieties into the ligand structure with the same features for anchoring the media, ${ }^{3}$ for example fluorinated ligands in organofluorinated solvents, ${ }^{\mathbf{4}, \mathbf{5}}$ polyethyleneglycol (PEG)-substituted ligands in PEG media ${ }^{6-8}$ and charged ligands in highly polar solvents. ${ }^{9-11}$

${ }^{a}$ Laboratory of Molecular Catalysis, Institute of Chemistry - UFRGS, Av. Bento Gonçalves 9500, 91501-970, CP 15003, Porto Alegre, RS, Brazil. Fax: +55(51) 33087304

${ }^{b}$ School of Chemistry, University of Nottingham, University Park, Nottingham, NG7 2RD, UK. E-mail: jairton.dupont@ufrgs.br
The use of charged ligands has attracted special attention, because water can be used as an anchoring medium, thereby combining sustainability and efficiency. ${ }^{11,12}$ The first successful protocol was the application of monosulphonated phosphines in rhodium-catalysed propene hydroformylation. ${ }^{\mathbf{1 3 - 1 5}}$

With the emergence of ionic liquids (ILs), the insertion of an ion-tagged side chain into the molecular skeleton of a known ligand became a useful protocol for anchoring ligands, and consequently catalysts, in an IL phase. ${ }^{\mathbf{1 6}}$ These ligands are referred to as ionophilic ligands or charge-tagged ligands (CTLs). The ionic modification confers a particular solubility profile that makes catalyst/product recovery possible, and often improves the activity of the catalytic species compared to the parent tag-free analogue. ${ }^{17}$ Moreover, new selective processes can be envisaged by changing the solubility and diffusion of substrates and products in the active phase of a reaction. ${ }^{18}$

In addition to the change in catalyst partition, the insertion of a cationic moiety into various reagents represents a powerful tool that can be used to detect reaction intermediates in organometallic catalysis through electrospray ionisation mass spectrometry (ESI-MS) experiments. ${ }^{19}$ Taking into account the
Jones Limberger obtained his Ph.D. degree from Universidade Federal do Rio Grande do Sul (UFRGS) in 2012 under the advice of Professor Adriano L. Monteiro. He is currently a postdoctoral fellow in Laboratory of Molecular Catalysis (Porto Alegre, Brazil), under the supervision of Professor Jairton Dupont. His current research interests focus on the development of novel metal-catalyzed methodologies for the obtainment of fine chemical products.
Bárbara C. Leal received her Bachelor degree in chemistry from UFRGS. In 2012, she completed her MSc at UFRGS under the supervision of Professor Jairton Dupont, focusing on the stabilization of palladium nanoparticles by ionophilic ligands in ionic liquids. Currently, she is a Ph.D. student at the same university in Prof. Dupont's research group. Her research interests are centered on catalysis using organometallic complexes and transition metal nanoparticles in ionic liquids. 
fact that ESI constitutes a mild ionisation technique, some neutral intermediates may not be ionised and, therefore, are not detected by MS. ${ }^{19}$ To overcome the detection problem of these "ESI-MS-blind intermediates", CTLs have been used. ${ }^{20,21}$ The insertion of an ionic tag ensures that the charge of an intermediate is not caused by the ESI. For this reason, these ligands have been used as ionic probes in mechanistic studies for several catalytic reactions. Scheme 1 illustrates the application of CTLs for both polar phase anchoring and ESI-MS detection.

There are extensive reviews covering both ESI-MS detection and the anchoring processes; a review of catalysts with ionic tags and their use in ILs was published in $2008 .{ }^{16}$ In the same year, a perspective review of using charged ligands for catalyst immobilisation and analysis appeared. ${ }^{21}$ In addition, it was shown that the use of a proper ionic tag can improve the performance of catalytic systems. ${ }^{22}$ Herein, we summarise examples from our laboratory and, where appropriate, recent literature studies on the use of CTLs as immobilising agents in organometallic catalysis and as probes for studying mechanisms through ESI-MS.

\section{CTLS as immobilising agents for organometallic catalyst} precursors

Olefin metathesis reactions. Our group has dedicated the recent years to synthesising imidazolium-based metathesis
CTLs. We have used these ligands to immobilise homogeneous catalysts in ILs. CTLs 1 and 2, for example, were easily obtained using three-step routes. These ligands were reacted with a second-generation Grubbs catalyst to produce the ionophilic Hoveyda-type complexes $\mathbf{3}, 4$ and 5 (Scheme 2). ${ }^{23}$ These complexes were successfully applied to ring-closing metathesis (RCM) reactions. Initially, it was observed that a judicious choice of anchoring IL is crucial for both activity and reusability. With the optimised conditions $\left(3, \mathrm{BMI} \cdot \mathrm{PF}_{6}(\mathrm{BMI}=\right.$ 1-butyl-3-methylimidazolium), $45{ }^{\circ} \mathrm{C}$, toluene co-solvent), turnover frequencies (TOFs) of up to $343000 \mathrm{~h}^{-1}$ were obtained in the 1,7-octadiene $\mathrm{RCM}$ reaction, even at ppm concentration
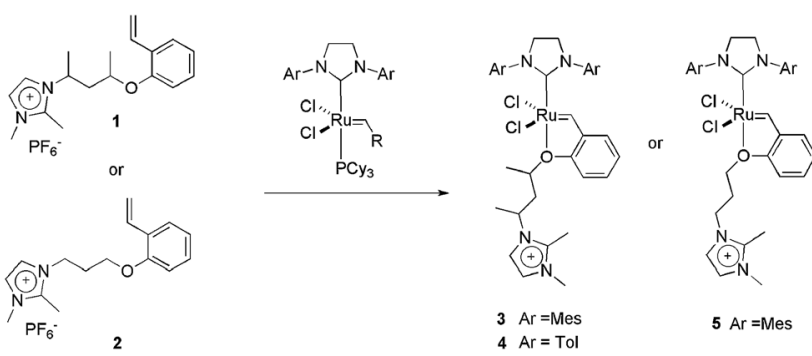

Scheme 2 Structure of CTLs 1 and 2 and the synthesis of chargetagged Hoveyda-type catalysts 3, 4 and 5 .
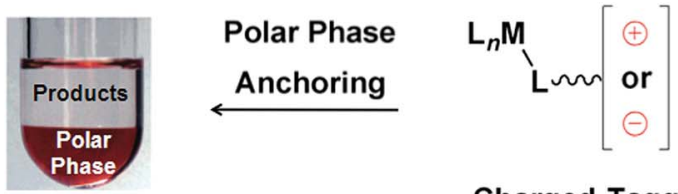

Charged-Tagged

Ligand

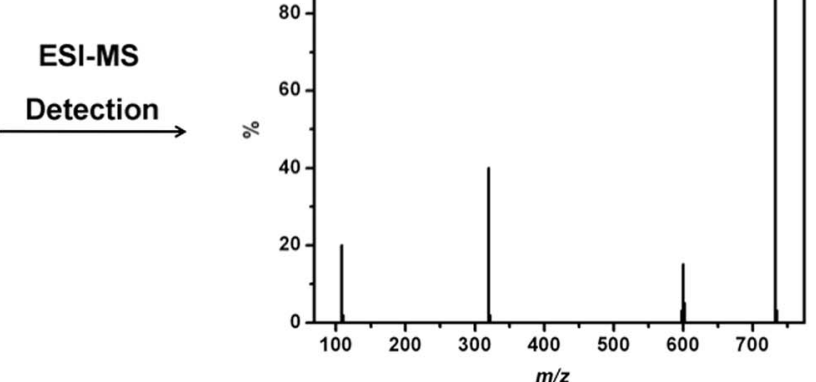

Scheme 1 CTLS for polar phase anchoring and ESI-MS detection.

Adriano L. Monteiro obtained his Ph.D. degree from the Université Paul Sabatier (Toulouse, France) in 1993 working with Dr Igor Tkatchenko, and was a postdoctoral fellow in 1998 at Massachusetts Institute of Technology (Cambridge, USA) working with Professor Stephen L. Buchwald. He joined the Department of Organic Chemistry of UFRGS (Porto Alegre, Brazil) in 1994 as assistant professor and is currently associate professor of Organic Chemistry. His research focuses on homogeneous catalysis with an emphasis on metal-mediated synthetic methodologies for the construction of $C-C$ bonds.
Jairton Dupont received his PhD at the Université Louis Pasteur of Strasbourg (France) and after a period as a post-doc at the University of Oxford (UK), he became a Professor of Chemistry at the Institute of Chemistry, UFRGS (Brazil). In 2014, he was appointed EPSRC/GSK Chair in Sustainable Chemistry at the School of Chemistry of the University of Nottingham (UK). He has been an invited Professor at various European Universities. He is a member of the Brazilian Academy of Sciences and the World Academy of Sciences; among the various distinctions he has received are the Humboldt Award, the Conrado Wessel Science Award, the TWAS Chemistry Award, the WIPO Award and the Brazilian Gran Cruz. His research interests are mainly centred on ionic liquids with special emphasis in catalysis, nanomaterials and alternative energies. 
levels. The reusability of the system was tested in the RCM of diallyldiethylmalonate, and seven cycles could be performed without an apparent decrease in activity. Another noteworthy finding is the fact that a high degree of substitution at the carbon attached to the oxygen in the ether portion of the CTL is beneficial to the catalytic activity.

We also successfully used CTL 3 in a tandem isomerisationmetathesis reaction of trans-3-hexene, affording heavier linear olefins. ${ }^{18}$ The reaction was performed in a biphasic system composed of $\mathrm{BMI} \cdot \mathrm{PF}_{6}$ and toluene. In this way, the metathesis catalyst, 1, was anchored in the IL phase and the isomerisation catalyst, RuHClCO $\left(\mathrm{PPh}_{3}\right)_{3}$, was kept in the organic phase. The use of this biphasic system is essential for keeping a superior olefin concentration in the toluene phase and, consequently, ensuring the correct isomerisation-metathesis rate. In this way, $80 \%$ of the initial trans-3-hexene was converted to an olefinic $\mathrm{C} 4-\mathrm{C} 17$ mixture, with $49 \%$ of the linear olefins being higher than C6.

As well as Hoveyda-type catalysts, we have also produced the charge-tagged Grubbs-type catalyst 6 . In this case, the ionic tag was provided by the ionophilic phosphine 7 . The synthesis of this CTL is easy, and could be achieved through a one-step radical chain addition of secondary phosphines to allyl imidazolium salts. ${ }^{24}$ The charge-tagged ruthenium complex 6 was applied to the RCM of 1,7-octadiene. When this reaction was performed in $\mathrm{BMI} \cdot \mathrm{PF}_{6}$-toluene, it afforded cyclohexene in a $98 \%$ yield with only 0.25 mol\% of the catalyst. Moreover, this system could be recycled eight times without loss of activity. This reusability was superior to that of the second-generation Grubbs catalyst, which exhibited a pronounced decrease in activity in the second cycle. Atomic absorption analysis of the toluene phase after each cycle, when using complex 6 , revealed a ruthenium content below the detection limits of the technique ( $<2$ ppm, i.e., less than $1.5 \%$ of the initial $\mathrm{Ru}$ content). Unfortunately, low conversions were observed when the system was applied to the RCM of highly substituted dienes. Fig. 1 illustrates the distribution of $\mathbf{6}$ and $\mathbf{8}$ (Grubbs catalyst) over the IL and toluene phases. Note that the toluene phase is uncoloured when using complex 6.

In 2010 , a fashionable system for the recovery of ruthenium metathesis catalysts was described using ligand $9{ }^{25}$ This ligand, when reacted with a second-generation Grubbs catalyst, produces the Hoveyda-type light-controlled charge-tagged

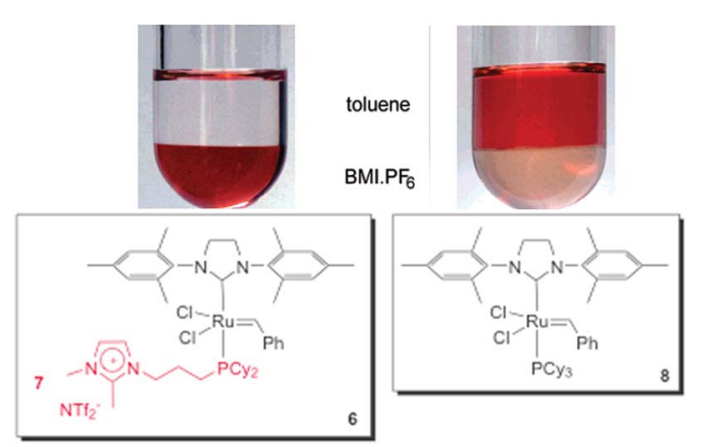

Fig. 1 Comparison of the distribution of 6 and 8 in a biphasic system composed of $\mathrm{BMI} \cdot \mathrm{PF}_{6}$ and toluene. Adapted from ref. 24. Copyright (2008) American Chemical Society. complex 10, which is a very active catalyst in the RCM of N-, Sand $\mathrm{O}$-containing substituted dienes (yields ranging from 90 to 97\% were obtained), even at low catalyst loadings. Moreover, this type of light-controlled tagged ligand can be switched between a non-polar and a polar phase through a tag-centred photoreaction. The photoreaction is reversible and results in drastic changes in the polarity and solubility of the ligand. In this way, after the RCM reaction, the solvent could be evaporated and the residue dissolved in a mixture of cyclohexane and alcohols $(1: 1)$. Thus, the catalyst and products were dissolved in the upper (cyclohexane) layer. The system was then irradiated with light $(\lambda>380 \mathrm{~nm})$ to transform the spiro form, 10, into the ionic tag 11, which completely shifted into the lower layer (alcoholic phase). The products remained in the apolar phase, which was subsequently removed. Next, $\mathrm{CH}_{2} \mathrm{Cl}_{2}$ was added and the mixture was kept in the dark for 3-5 $\mathrm{min}$ in order to reobtain the neutral species 10 (Scheme 3). The solvent mixture was then evaporated and $\mathrm{CH}_{2} \mathrm{Cl}_{2}$, in conjunction with a new bath of diene, was added; six RCM cycles could be performed, retaining almost the same activity.

Recently, ruthenium supported ionic liquid phase (SILP) catalysts based on alginates or chitosans were synthesised and applied in olefin metathesis reactions with a high level of recyclability and reusability combined with a good reactivity. ${ }^{26}$ The CTL 12 and the ruthenium supported ionic liquid catalyst (Ru bio-SILP) used in this work are illustrated in Scheme 4. The reactions were performed under both mono- and biphasic conditions using the ionic liquid $\mathrm{BMI} \cdot \mathrm{PF}_{6}$. The best results were obtained in the RCM of diethyl-2,2-diallylmalonate under heterogeneous conditions with the alginate based $\mathrm{Ru}$ bio-SILP (at least 15 cycles could be performed with high activity $\geq 87 \%$ ). The detection of ruthenium leaching by ICP-MS in the extraction phases showed low leaching of Ru throughout the cycles (200 ppm). This system was also active in the cross-metathesis reaction, with a high level of reusability (at least 12 cycles with activities up to $90 \%$ ). This reusability could be attributed to the good anchoring of the catalyst in the IL phase supported on the biopolymer.

Metal-catalysed cross-coupling reactions. Several examples of using substituted imidazolium salts as both the solvent and the ligand in cross-coupling reactions have been reported (Fig. 2). In these CTLs, a coordinating imidazole, pyrazole or pyridine group was attached to either $\mathrm{C} 2$ or $\mathrm{C} 1$ of the imidazolium moiety. ${ }^{27-30}$ For instance, mono-charged ligand 13, in combination with $\mathrm{PdCl}_{2}$, was very active in both Suzuki (yields

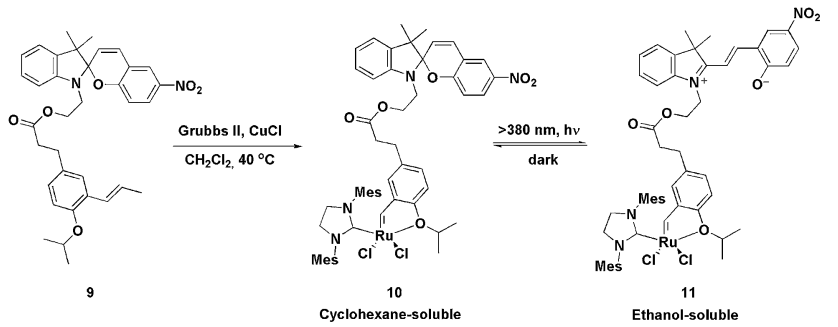

Scheme 3 Synthesis and photoreaction of the spiro form of tagged ruthenium-photo-controlled complex 10 . 


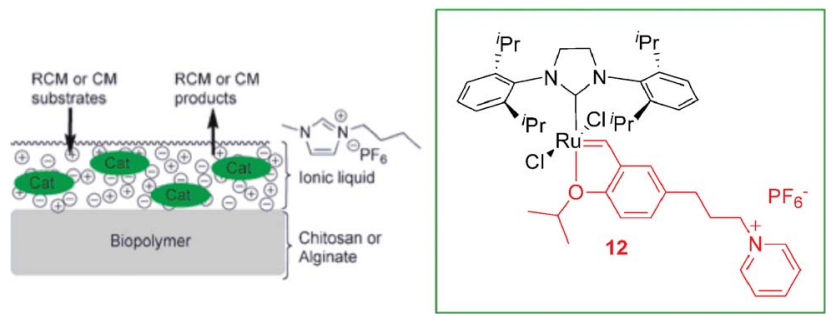

Scheme 4 Schematic representation of the ruthenium bio-SILP. Adapted from ref. 26.

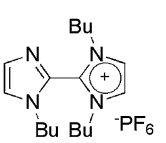

13

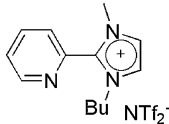

14

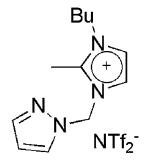

15

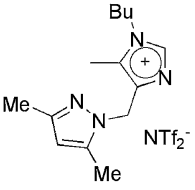

16
Fig. 2 CTLs used as the solvent/ligand in palladium-catalysed crosscoupling reactions.

ranging from 80 to $90 \%)^{29}$ and Heck (yields ranging from 70 to $94 \%$ ) cross-couplings. ${ }^{28}$ In terms of reusability, for the Heck reaction, the system could be reused 9 times, even when less active aryl chloride was used. For the Suzuki cross-coupling reaction, the system could be reused 14 times without an apparent loss of activity.

The complex resulting from the reaction of 14 and $\mathrm{PdCl}_{2}$ was used in the Heck reaction of acrylates and styrenes with aryl iodides or bromides, using 14 as the solvent. ${ }^{27}$ In the reaction of PhI with $n$-butyl acrylate, a 99\% yield was obtained over nine Heck reaction cycles. Moreover, yields from 49 to $94 \%$ were obtained when the same catalytic system was reused 14 times whilst varying the coupling partners.

Pyrazolyl-substituted ionophilic compounds 15 and 16 were effective when used as the solvent and ligand to stabilise the palladium species in Heck, Suzuki and Sonogashira crosscoupling reactions. ${ }^{30}$ In all cases, the catalysts could be reused several times without loss of activity.

In addition to nitrogen-based ligands, phosphorus CTLS have also been effectively employed in palladium-catalysed cross-coupling reactions. The tagged diphenylphosphine ligand 17, for example, was used to produce palladium complex 18 (Scheme 5). ${ }^{31}$ This precursor was active in the Suzuki coupling of aryl bromides and arylboronic acids in a mixture of 1-butyl-1methyl-pyrrolidinium bis(trifluoromethylsulfonyl)imide and water $(2: 1)$. With a catalyst loading of $1 \%$, the biaryls were

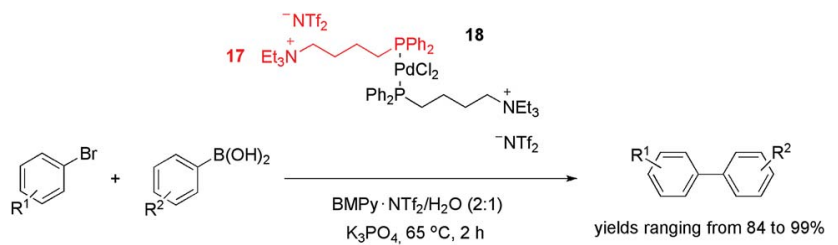

Scheme $5 \mathrm{PdCl}_{2}$-tagged phosphine used as a precursor in the Suzuki cross-coupling reaction. obtained with yields ranging from 84 to 99\%. Surprisingly, when aryl iodides were used, lower yields were obtained. This result was attributed to IL anion exchange (from $\mathrm{NTf}_{2}{ }^{-}$to $\mathrm{I}^{-}$). This hypothesis was supported by the fact that the addition of $\mathrm{KI}$ inhibited the conversion, whereas the addition of $\mathrm{KBr}$ did not cause a significant decrease in the reaction rate. In terms of reusability, six cycles could be performed without an apparent loss of activity. Moreover, no "Pd black" was observed, and $<10$ $\mathrm{ppb}$ of the metal was leached from the IL into the product during recycling.

It is important to note that, in the majority of these cases, the use of "activated" aryl iodides and bromides suggests the probable involvement of $\operatorname{Pd}(0)$ species, including low nuclearity Pd nanoparticles (NPs). ${ }^{32}$ Moreover, in the cases involving the imidazolium nucleus, it is quite probable that $\mathrm{N}$-heterocyclic (NHC) carbenes are involved, at least as transient species. ${ }^{33}$ However, in each of these cases, the CTL is able to stabilise and retain the active catalytic species in the IL phase.

Another important aspect of Heck coupling reactions in ILs is the possibility to induce a higher degree of selectivity, because the neutral and ionic pathways of this reaction can lead to opposite regioselectivities. Indeed, the Pd complexes associated with P-containing ligands in $\mathrm{BMI} \cdot \mathrm{BF}_{4}$ promote the regioselective arylation of butyl vinyl ether, affording the $\alpha$-isomer almost exclusively. Conversely, reactions performed in organic solvents afford variable mixtures of the $\alpha$ - and $\beta$-isomers. ${ }^{34}$ Unfortunately, this process has not yet been investigated using CTLs.

A variety of CTLs/ILs has also been used to promote better NP stabilisation, as well as to extend the catalyst's lifetime during catalytic reactions. In this context, the catalytic activity of palladium complexes immobilised in alkyl-substituted and nitrilefunctionalised pyridinium ILs was tested in the coupling of tributylphenylstannane and iodobenzene. ${ }^{35}$ However, the presence of $[\operatorname{Pd}(0)]_{n}$ NPs was identified after the Stille reaction. Moreover, recycling experiments showed that the functionalised IL was a superior reaction medium compared with a non-functionalised analogue, which may be associated with enhanced stabilisation of the formed NPs in the nitrile-pyridinium CTL/IL, as corroborated by transmission electron microscopy (TEM). Imidazolium-based CTL/ILs containing nitrile groups attached to the alkyl side chain were also employed in the Stille reaction (Scheme 6). ${ }^{36}$ In this case, a detailed study showed the influence of the relative coordination strengths of the cations and anions on the efficiency of the coupling reaction. Interestingly, NPs were only formed in some cases, and the superior conversion observed for the nitrile-functionalised IL compared with the non-functionalised one was assigned to the improved stabilisation of the NPs offered by the nitrile CTL.

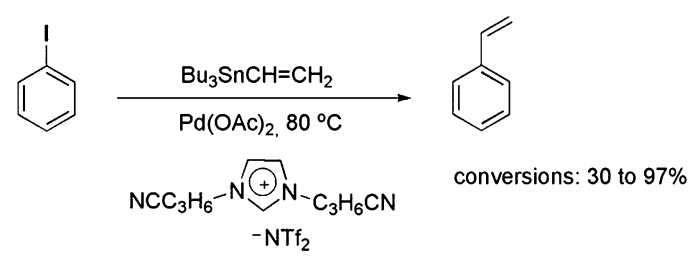

Scheme 6 Stille reaction and the IL/ligand that was employed. 
A novel bisphenol-functionalized benzimidazolium salt was synthesised and applied in the obtainment of the air-stable ionic iron(III) complexes 19 and 20 with 85 and 82\% yields, respectively (Scheme 7). ${ }^{37}$ Iron-based catalysts have been employed in the alkylation of Grignard reagents ${ }^{38,39}$ in place of palladium and nickel complexes, because of the possibility of undesired hydride $\beta$-elimination in these complexes. However, in most reports, the more active alkyl bromides and iodides are employed as the electrophilic partner using catalyst loadings around 5 mol\%. Both complexes 19 and 20 afforded crosscoupling products with reasonable to excellent yields using 1 mol\% of iron and secondary alkyl chlorides as the electrophile.

On the other hand, the results using primary chlorides were unsatisfactory, considering that yields ranging from 5 to $45 \%$ were observed. In recycling experiments, 19 provided the coupling product with yields greater than 95\% for six runs, however, in these experiments $5 \mathrm{~mol} \%$ of iron (instead of 1 mol\%) and a more active alkyl bromide were applied. ${ }^{37}$ The authors also highlighted that 19 outperforms the catalytic activity of an imidazolium analogue, ${ }^{\mathbf{4 0}}$ especially in avoiding the formation of the $\beta$-elimination product.

Metal-catalysed enantioselective reactions. Several examples of enantioselective modifications have been reported that were catalysed by complexes with ionically tagged ligands. For example, an imidazolium cation bound to an $\eta 6$-arene ruthenium chiral complex was evaluated and compared to neutral $\eta 6$ $p$-cymene complexes in hydrogenation transfer reactions. ${ }^{\mathbf{4 1}}$ Acetophenone was used as the substrate and 2-propanol or formic acid was used as the proton source (Scheme 8). For 21, the enantiomeric excess (ee) was similar to the neutral counterpart (98\%). In addition, for both $\mathbf{2 1}$ and 22, the leaching was lower than for the neutral complexes when the reaction was performed in 1-butyl-2,3-dimethylimidazolium hexafluorophosphate. However, the reaction yield decreased upon recycling. This result was attributed to base-induced catalyst degradation.

Imidazolium-tagged ferrocenyl diphosphines $\mathbf{2 3}$ and $\mathbf{2 4}$, analogues of Josiphos ligands, were employed in palladiumcatalysed enantioselective allylic substitution reactions with heteroatom nucleophiles (Scheme 9); ${ }^{\mathbf{4 2}}$ potassium phthalimide, 4-methylphenolate and $p$-tolylsulfinite were also employed. For the N-containing nucleophile, the most favourable compromise

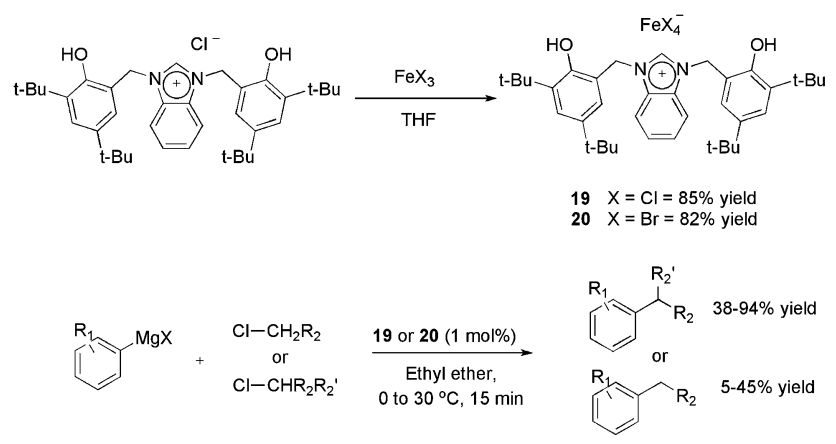

Scheme 7 Synthesis of ionic iron-benzimidazole complexes and their application in the cross-coupling of aryl-Grignard reagents with alkyl halides.

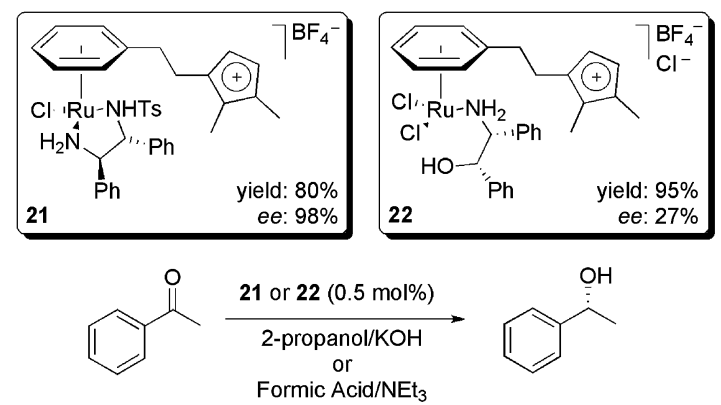

Scheme 8 lonophilic ruthenium complex-catalysed transfer hydrogenation reactions.

between yield and enantioselectivity was obtained in a medium composed of $\mathrm{EBI} \cdot \mathrm{EtOSO}_{3}$ and $\mathrm{CH}_{3} \mathrm{CN}$ (70\% yield and $92 \%$ ee).

For O- and S-containing nucleophiles, only modest results were obtained (41\% yield with $62 \%$ ee and $81 \%$ yield with $10 \%$ ee, respectively). Moreover, when recycling experiments were performed, disappointing results were obtained. This was attributed to the high reactivity of the $\mathrm{C}-\mathrm{H}$ bond at the $\mathrm{C} 2$ position in the imidazolium moiety of $\mathbf{2 3}$ under basic conditions. However, when CTL 24, with a methylated C2, was employed, no improvements were achieved in yield or enantioselectivity.

Recently, allyl-palladium complexes containing imidazolium-tagged chiral diaminophosphite ligands (S,S)-25 and (R)26 were synthesised and also applied as catalysts in asymmetric allylic substitution reactions with heteroatom and carbon nucleophiles (Scheme 10). ${ }^{43}$ Complexes containing one and two units of $(\mathrm{S}, \mathrm{S})-25 /(\mathrm{R})-26$ were tested in $\mathrm{BMI} \cdot \mathrm{PF}_{6}$ and $\mathrm{BMPyr} \cdot \mathrm{NTf}_{2}$ as reaction media. Only $1 \mathrm{~mol} \%$ of palladium was enough to promote the substitution with up to $100 \%$ conversion for $\mathrm{N}$ and $\mathrm{C}$ nucleophiles and 85\% conversion for S nucleophiles, in three hours at $35{ }^{\circ} \mathrm{C}$. Concerning the enantioselectivity, $74 \%$ ee was obtained using benzylamine, $72 \%$ ee was observed for sodium $p$-toluenesulfinate and only $45 \%$ ee was obtained using sodium diethylmalonate. The best results, considering both reactivity and enantioselectivity, were attained using [ $\mathrm{PdCl}($ methallyl)26] $\left[\mathrm{BF}_{4}\right]$ in $\mathrm{BMPyr} \cdot \mathrm{NTf}_{2}$. In recyclability tests, unimpressive results were observed, since the conversion decreased from 75 to $55 \%$ in four runs, while the ee decreased from 75 to $58 \%$ ee over 10 reaction cycles. These results were attributed to catalyst

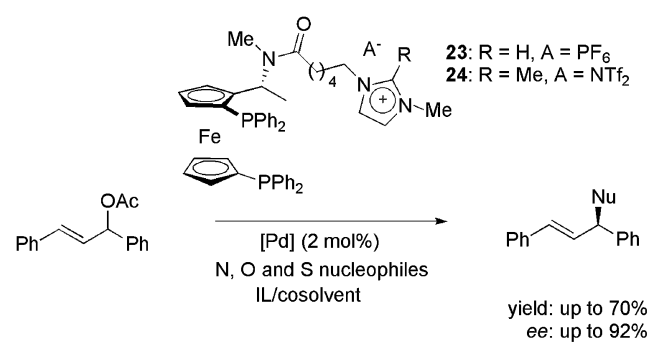

Scheme 9 Imidazolium-tagged ferrocenyl diphosphines employed in the palladium-catalysed allylic substitution with heteroatom nucleophiles. 

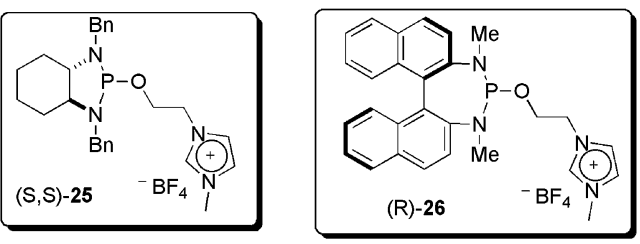

$\mathrm{Pd}$ (methallyl $\left.) L_{2}\right]\left[\mathrm{BF}_{4}\right]_{3}$

$$
\text { or }
$$
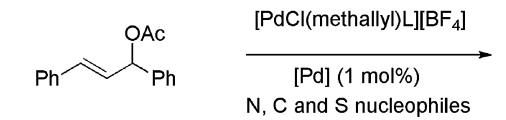

$\mathrm{N}, \mathrm{C}$ and $\mathrm{S}$ nucleophiles
$\mathrm{BMI} \cdot \mathrm{PF}_{6}$ or BMPyr$\cdot \mathrm{NTf}_{2}$

$L=(S, S)-25,(R)-26$

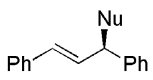

Conversion: $85-100 \%$

ee: $45-74 \%$
Scheme 10 Application of allyl-palladium complexes containing an imidazolium-tagged chiral diaminophosphite ligand in N, C, and S asymmetric allylic substitution.

deactivation, given that palladium was not detected in the ICPMS analyses of the organic extractive phase. It is important to mention, however, that to the best of our knowledge, only one efficient IL-based recycling system has been reported for Pdcatalysed asymmetric allylic substitution. ${ }^{\mathbf{4 4}}$

Ionically tagged Josiphos analogues, 27, were applied in the rhodium-catalysed hydrogenation of the benchmark substrates methyl acetamidoacrylate (MAA) and dimethyl itaconate (DMI) (Scheme 11). ${ }^{45}$ The cationic precursor, $\mathrm{Rh}(\mathrm{NBD}){ }_{2} \mathrm{BF}_{4}(\mathrm{NBD}=$ 2,5-norbornadiene), was used with a $\mathrm{H}_{2}$ pressure of 1 bar at room temperature. Initially, it was observed that the use of the appropriate reaction media was crucial for both activity and enantioselectivity. By using a biphasic system composed of tertbutyl methyl ether and $\mathrm{BMI} \cdot \mathrm{BF}_{4}$, total conversion and a $99 \%$ ee were obtained. In the recycling experiments, the ionic ligands kept the TOF above $1000 \mathrm{~h}^{-1}$ for at least five cycles, whereas with neutral Josiphos ligands the reaction rate decreased markedly upon each reuse.

A series of ionophilic monophosphites, 28, based on Dmannitol was synthesised and successfully applied in the rhodium-catalysed, enantioselective hydrogenation of enamides, dehydroamino acids and dimethyl itaconate (Scheme 12). ${ }^{46}$ The ligands provided high yields and excellent enantioselectivity in either the $\mathrm{CH}_{2} \mathrm{Cl}_{2}$ or biphasic IL/toluene media. The best results were obtained with the ligand bearing a sixcarbon alkyl chain between the mannitol and imidazolium moieties. The recyclability of the system was tested in the

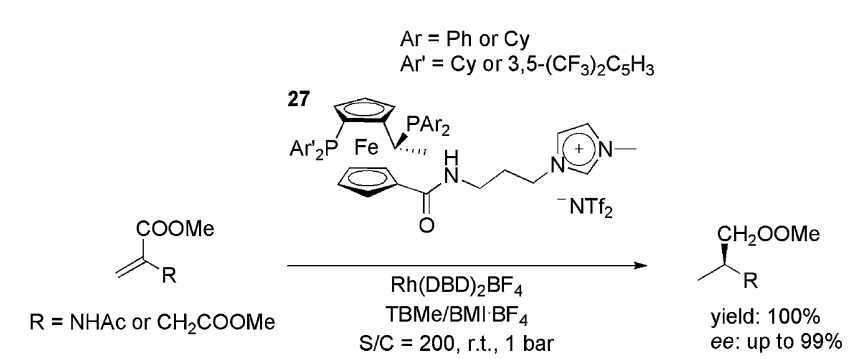

Scheme 11 Cationic modified Josiphos ligands that were employed in rhodium-catalysed, biphasic, enantioselective hydrogenation.

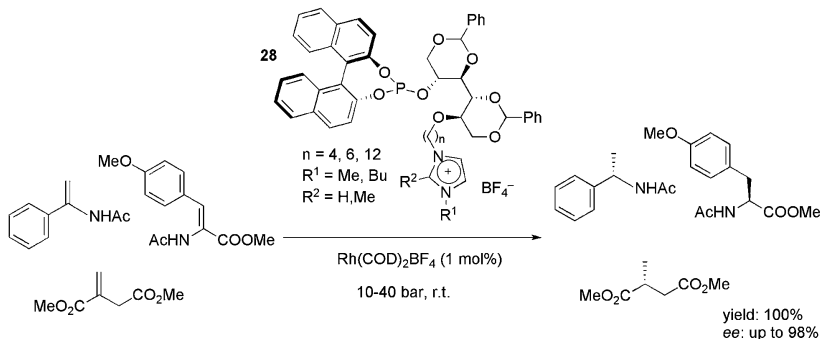

Scheme 12 Mannitol-based charge-tagged monophosphites that were used as ligands in the rhodium-catalysed hydrogenation of olefins.

$\mathrm{BMI} \cdot \mathrm{BF}_{4}, \mathrm{BMI} \cdot \mathrm{PF}_{6}$ and $\mathrm{BMMI} \cdot \mathrm{BF}_{4}(\mathrm{BMMI}=1$-butyl-2,3-dimethylimidazolium) ILs. In all cases, up to eight recycles could be performed, keeping the yield and enantioselectivity almost constant.

In 2012, the use of the imidazolium-tagged bis(oxazoline) ligand 29 was reported in the copper-catalysed, asymmetric Henry reaction (Scheme 13). ${ }^{\mathbf{4}}$ Several substituted benzaldehydes were reacted with $\mathrm{CH}_{3} \mathrm{NO}_{2}$, providing the corresponding adducts. The reaction was effective in various ILs and organic solvents. However, the best enantioselectivity was obtained when the reaction was performed in $\mathrm{MeOH}$. In this medium, 23 to $94 \%$ ee was obtained. Moreover, the catalyst could be reused six times, by evaporating the solvent and extracting the product with ethyl ether, without loss of activity or enantioselectivity.

Metal-catalysed oxidation of olefins. An ionophilic iron(III) complex, 30, bearing three imidazolium moieties was synthesised (Scheme 14) by reacting 31 and $\mathrm{FeCl}_{3}$ in methanol. ${ }^{\mathbf{4}}$ This tagged complex was used in the epoxidation of methyl oleate and vegetable oils. Yields ranging from 79 to $98 \%$ were obtained when the reactions were performed in $\mathrm{BMI} \cdot \mathrm{NTf}_{2}$, using synthetic air at 15 bar as the oxidising agent. In the oxidation of methyl oleate, the catalytic system was recycled ten times without an apparent decrease in activity. Moreover, no significant iron leaching $(<2 \mathrm{ppm})$ was observed by means of inductively coupled plasma atomic emission spectroscopy (ICP-AES).

The use of ionophilic manganese porphyrins (Fig. 3) in alkane and alkene oxidation reactions has also been reported. Pyridinium-substituted catalysts 32 and 33, for instance, were applied to ethylarene oxidation (Scheme 15). ${ }^{49}$ Under optimised conditions $\left(0.4 \mathrm{~mol} \%\right.$ of $32, \mathrm{BPy} \cdot \mathrm{BF}_{4}$, and $\mathrm{PhIO}$ as the oxidising

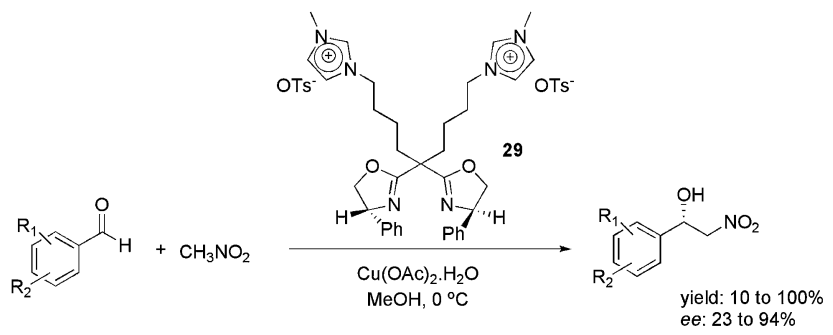

Scheme 13 Imidazolium-tagged bis(oxazoline) ligand that was used in the copper-catalysed, asymmetric Henry reaction. 


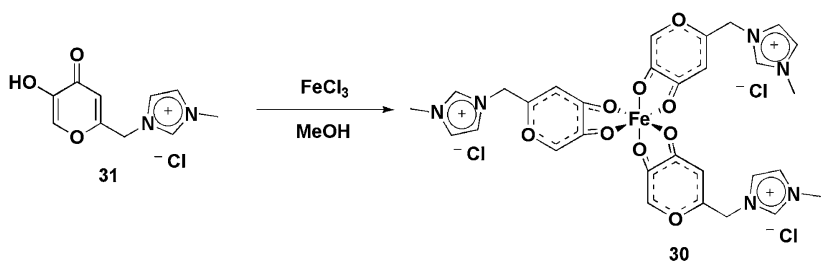

Scheme $14 \mathrm{Fe}(\mathrm{II})$ complex bearing imidazolium moieties that was applied in the olefin epoxidation reactions.

agent) ethylbenzene was oxidised to 1-phenylethanol and acetophenone with 71 and $29 \%$ selectivity, respectively. In recycling experiments, for both $\mathbf{3 2}$ and 33, the selectivity for the ketone increased with successive reuse. In terms of activity, for 32 , the conversion decreased with reuse, whereas for 33 it remained constant over four oxidation cycles. When substituted arylethenes were used as substrates, a positive effect on the conversion was observed for the electron-donating groups. According to the authors, this result is due to the increased electron density at the $\alpha$-carbon of ethylbenzene derivatives.

Catalyst 32 was also used in the oxidation of arylethenes in $\mathrm{CH}_{3} \mathrm{CN}$ and $\mathrm{BPy} \cdot \mathrm{BF}_{4}{ }^{.0}$ The system was active and selective for the formation of epoxides, in both media. Moreover, the recyclability of $\mathbf{3 2}$ was superior to that of the non-ionophilic analogue.

The synthesis of manganese porphyrins, bound to one, three or four imidazolium units (34), has been described ${ }^{51,52}$ and applied to olefin epoxidation reactions, using $\mathrm{H}_{2} \mathrm{O}_{2}$ as the oxidizing agent (Scheme 15). In an early investigation, ${ }^{51}$ ligand 34c (olefin-catalyst $=75: 1$ ) was applied efficiently to the epoxidation of cyclooctene, cyclohexene, $1 H$-indene and

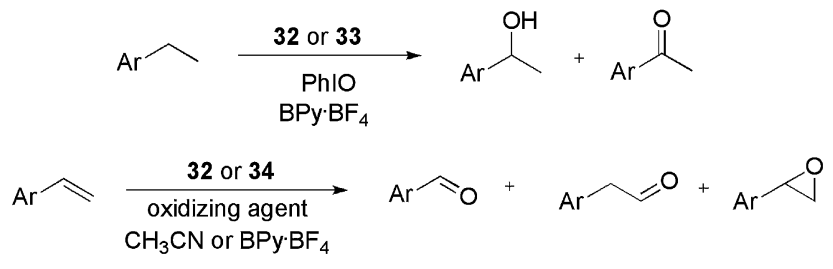

Scheme 15 Manganese porphyrins bound to imidazolium units, which were applied to the oxidation of olefins and alkanes.

styrene. The oxirane products were obtained with yields ranging from 95 to $100 \%$ in $\mathrm{CH}_{3} \mathrm{CN}$, with acetic acid as the co-catalyst. Next, charge-tagged metalloporphyrins were compared to the neutral analogue $\left[\mathrm{R}^{1}-\mathrm{R}^{4}=1,5-\mathrm{Cl}\left(\mathrm{C}_{6} \mathrm{H}_{3}\right)\right]$ in arylethene epoxidation..$^{52}$ All catalysts $(34 a-34 c)$ were active in the epoxidation of styrene with a $S / C$ ratio of 300 . High conversions were attained with catalysts 34b and 34c. However, the best selectivity was obtained with the mono-tagged complex 34a, which provided similar results to the neutral complex (selectivity $>90 \%$ ). By using substituted styrenes, the authors were able to obtain Hammett plots for the reaction. Again, the reaction was accelerated by electron-donating groups and, furthermore, the small $\rho$-values suggested no significant charge separation in the transition state.

Metal-catalysed olefin hydroformylation. The cationic phosphine CTL 35 was applied in the rhodium-catalysed tandem hydroformylation-acetalyzation of olefins in ionic liquidalcohol media (Scheme 16). ${ }^{53}$ Initially, 1-octene was used as the substrate model for the optimization of the ligand counteranion, catalyst loading, and ionic liquid medium. In this way, using $35 \cdot \mathrm{BF}_{4}$ and $0.1 \mathrm{~mol} \%$ of rhodium in a medium composed

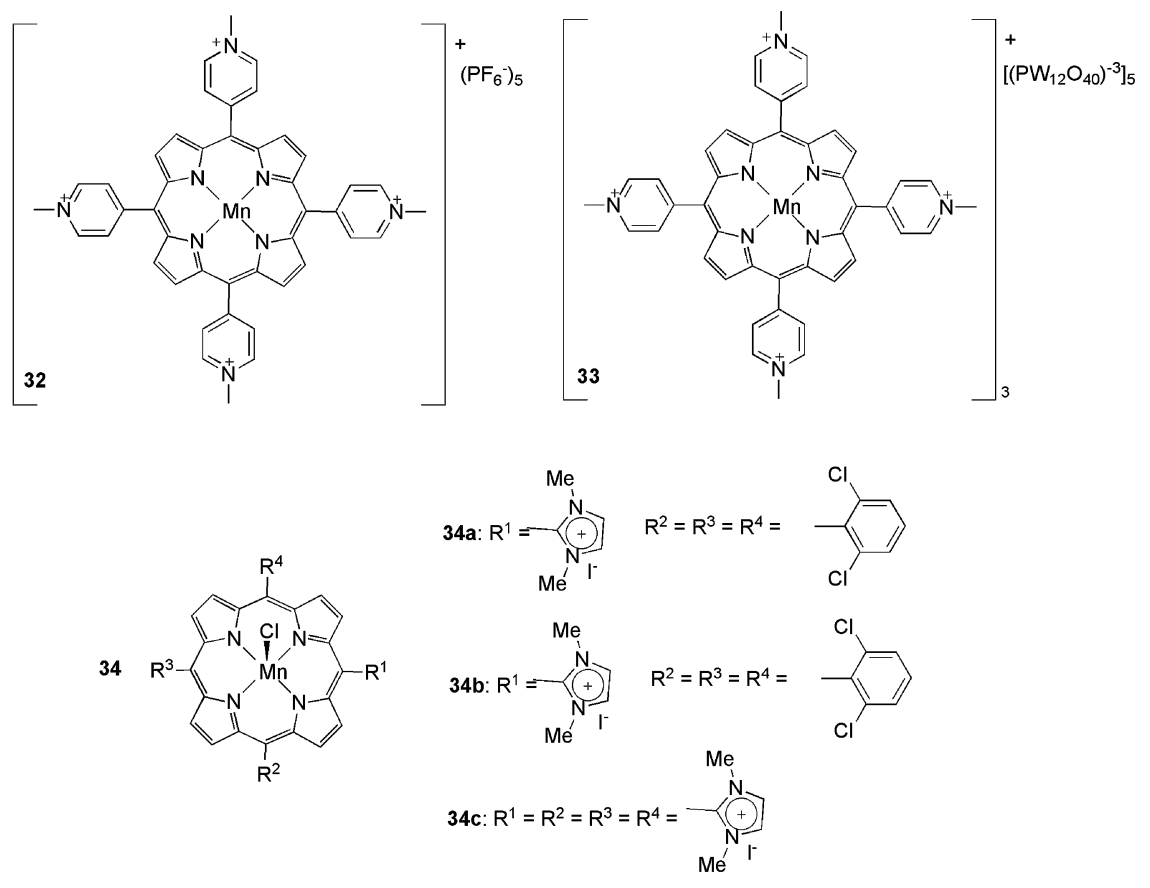

Fig. 3 Manganese porphyrins that were used in the oxidation reactions. 


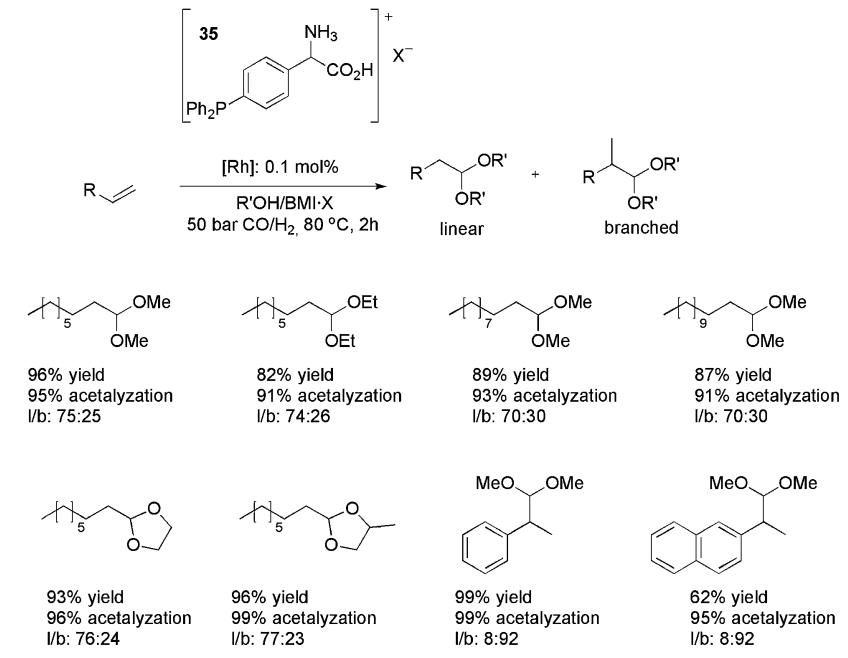

Scheme 16 CTL 35 applied in the rhodium-catalysed tandem hydroformylation-acetalyzation of olefins in ionic liquid/alcohol media.

of $\mathrm{BMI} \cdot \mathrm{BF}_{4}$ and methanol, the product of the hydroformylation was obtained in a 96\% yield, with $95 \%$ acetalyzation and a linear-branched ratio of $75: 25$ after $2 \mathrm{~h}$ at $80{ }^{\circ} \mathrm{C}$ and $\mathrm{CO}-\mathrm{H}_{2}$ $(1: 1)$ at 50 bar. Following this, other alkenes (alkyl and aryl substituted) were also hydroformylated in the presence of methanol, ethanol, ethylene glycol and propylene glycol, affording the products observed in Scheme 16. The high levels of acetalyzation were attributed to the Brønsted-acid character of the ligand 35 , since only $1 \%$ acetalyzation was observed when the CTL was substituted by $\mathrm{PPh}_{3}$. It is worth noting the recyclability results observed with this strategy. For a system composed of $\mathrm{Rh}(\mathrm{acac})(\mathrm{CO})_{2}, 35 \cdot \mathrm{BF}_{4}, \mathrm{BMI} \cdot \mathrm{BF}_{4}$ and methanol, conversions greater than $98 \%$ and an average acetalyzation of $95 \%$ were observed over 12 runs. When methanol was replaced by propyleneglycol, at least 15 runs were performed without significant loss of activity. With both alcohols, the lixiviation of rhodium from the IL phase was less than $0.1 \%$.

CTLs as immobilising agents in NP-catalysed hydrogenation reactions. Metal NPs have been widely used in catalysis because of their unique properties. However, it is well known that "naked" NPs are thermodynamically unstable and must be stabilised to prevent agglomeration. In this context, ionophilic ligands have been employed in catalysis to improve NP activity and stability and, most importantly, to immobilise these NPs in the ionic phase. ${ }^{54}$

The catalytic activity was strongly influenced by the structure of the CTL stabiliser employed in arene hydrogenation with $[\mathrm{Rh}(0)]_{n} \mathrm{NPs}$, following the trend $36>37>38$ (Scheme 17).$^{55}$ The $[\mathrm{Rh}(0)]_{n}$ NPs stabilised by 36, containing a C7 alkyl chain between the bipyridine and the imidazolium group, showed the highest activity compared to the ligand with only a methylenic group between the bipyridine and the ionophilic group 38. This may be caused by a weaker interaction between the ligand $\mathbf{3 8}$ and the NP surface, which decreases the binding affinity of this ligand and promotes the decomposition of the NPs under the reaction conditions.

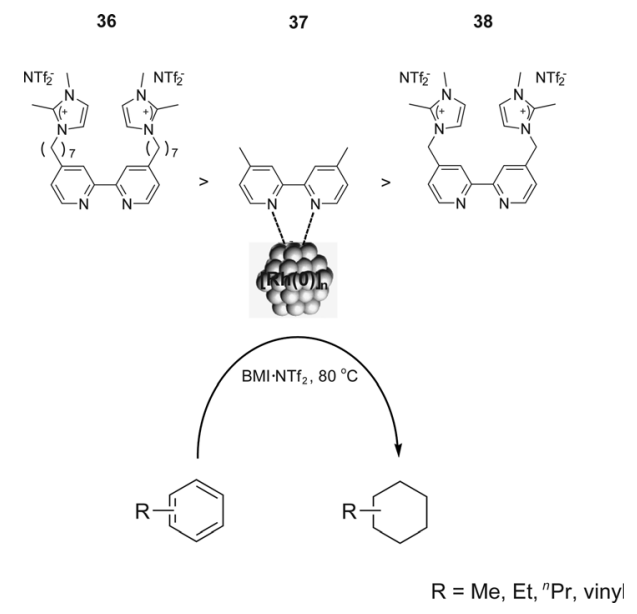

Scheme 17 Hydrogenation of arenes, catalysed by $[R h(0)]_{n}$ NPs stabilised by CTLs in ILs, and the activity observed for the different ligands.

Similarly, bipyridinium CTLs (39 and 40) were employed in styrene hydrogenation using $[\mathrm{Rh}(0)]_{n} \mathrm{NPs}^{56}$ The total conversion of styrene into ethylcyclohexane was observed and the quantitative hydrogenation of the aromatic ring was attributed to the monodentate coordination of the ligand to the NP (Scheme 18). Furthermore, NPs with small sizes (around 2.0$2.5 \mathrm{~nm}$ ) and good stabilities were obtained in the presence of charged ligands. Comparable results were obtained in arene hydrogenation using $[\mathrm{Rh}(0)]_{n}$ NPs stabilised by imidazoliumfunctionalised bipyridine derivatives in $\mathrm{BMI} \cdot \mathrm{PF}_{6} \cdot{ }^{57}$ Conversions of up to $85 \%$ were obtained for the total hydrogenation of benzene and toluene.

In the same context, functionalised ILs have been employed as the CTL/solvent in catalysis to improve the stability of the NPs. $[\mathrm{Ru}(0)]_{n}$ NPs synthesised in nitrile-functionalised ILs display unusual selectivities towards the hydrogenation of nitrile-containing aromatic compounds. ${ }^{58}[\mathrm{Ru}(0)]_{n} \quad$ NPs dispersed in $(\mathrm{BCN}) \mathrm{MI} \cdot \mathrm{NTf}_{2}[(\mathrm{BCN}) \mathrm{MI}=1$-butyronitrile-3methylimidazolium], 41, exclusively hydrogenate nitrile groups instead of arenes, which are typically hydrogenated by $[\mathrm{Ru}(0)]_{n}$ NPs in non-functionalised ILs $^{59}$ (Scheme 19). It is plausible that, in these systems, the nitrile group of the IL is strongly

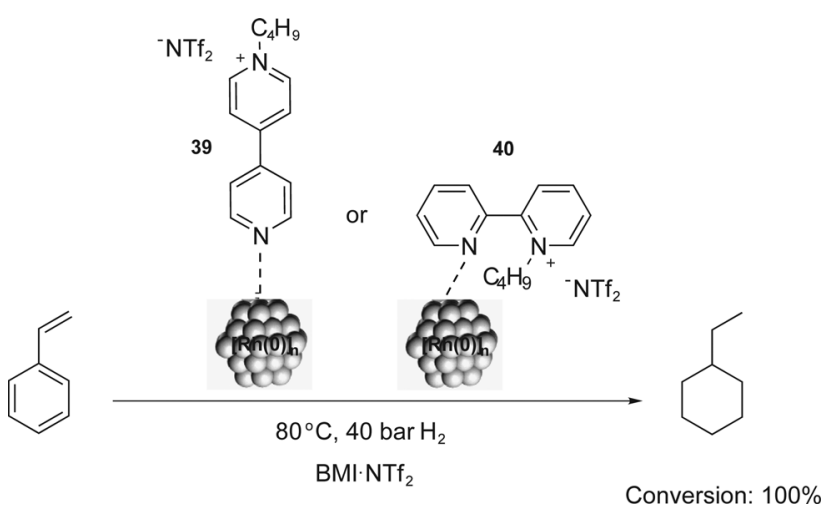

Scheme 18 Hydrogenation of styrene catalysed by $[\mathrm{Rh}(0)]_{n} \mathrm{NPs}$, which are stabilised by the bipyridinium CTLs 39 and 40 . 
coordinated to the ruthenium surface, which only allows the nitrile group of the substrate access to the NP surface, thereby avoiding arene coordination. Thus, it is possible to state that the presence of a CTL, effectively, modulates the selectivity of the catalyst.

The selective hydrogenation of alkynes to $(Z)$-alkenes, catalysed by $[\operatorname{Pd}(0)]_{n}$ NPs $(7.3 \mathrm{~nm})$ stabilised in the CTL/IL 41, was also recently reported by our group. ${ }^{60}$ Notably, 3-hexyne was partially hydrogenated to the corresponding ( $Z$ )-olefin without any trace of the isomerised by-product. The lack of isomerisation indicates that the $[\operatorname{Pd}(0)]_{n}$ NPs exhibit typical behaviour and possess surface-like (multi-site) catalytic properties. Moreover, it is possible to tune the selectivity towards alkenes or alkanes, depending on the hydrogen pressure employed (Scheme 20). These results are probably related to the low hydrogen gas solubility in ILs and the associated mass-transfer/ diffusion limitations. It is expected that, at low pressures, there is a minimal amount of hydrogen dissolved in the IL, affording the alkene product; on the other hand, increasing the gas pressure results in more hydrogen being present in the IL phase to yield total hydrogenation (i.e., the alkane product).

\section{CTLs as probes for detecting reaction intermediates through ESI-MS}

In the last decade, ESI-MS has become a fundamental technique for the detection of intermediates in organometallic catalysis. ${ }^{2,61-63}$ This method permits the transfer of ions from solution into the gas phase, thus allowing the sampling of dissolved, charged organometallics in situ. By using this methodology, important information concerning the catalytic cycle of a plethora of reactions could be obtained (for instance crosscoupling reactions, ${ }^{64-66}$ the Wacker oxidation, ${ }^{67}$ hydrosilylation, ${ }^{68}$ hydrogenation, ${ }^{69}$ etc.), including air- and moisturesensitive ones. ${ }^{70}$ However, ESI represents a "gentle" ionisation method, and only charged intermediates are detectable in ESIMS. Therefore, reactions that proceed through a neutral mechanism are not likely to be detected properly. Moreover, the common ionisation techniques, protonation/deprotonation, can affect the nature of the catalytic system under investigation. To overcome this limitation, some strategies have been employed, including (i) the formation of charged adducts ${ }^{71}$ and (ii) the use of charge-tagged substrates (this strategy has been applied successfully for olefin metathesis, ${ }^{20}$ cross-coupling reactions, ${ }^{19,72,73}$ the Pauson-Khand $[2+2+1]$ cycloaddition $^{74}$ and other catalytic processes ${ }^{75}$ ). However, both (i) and (ii) limit the scope of the substrates employed. An alternative is to use ionically marked ligands. Here, we describe interesting examples of the use of CTLs as probes to detect intermediates in catalysis. Note that although charged metal compounds behave straightforwardly in ESI-MS, low fragmentation conditions should be used i.e. a low cone angle voltage in order to observe parent ions or derivatives. For the details, scope and limitations of the use of ESI-MS readers are invited to consult the specialized literature. ${ }^{76-79}$

Olefin metathesis. The pioneering work on CTLs to be used in the detection of intermediates through ESI-MS was published by Chen and co-workers in $1998 .^{80}$ The authors used the charged-tagged ruthenium complex 42 as a probe in crossmetathesis (with 1-butene) and ROM (with cyclobutene, cyclopentene and norbornene) reactions (Scheme 21). Initially, the signal of $\mathbf{4 3}$ was observed in the gas phase, corresponding to the de-coordination of a phosphine ligand. Next, after the addition of the olefins, the metathesis adducts 44-46 were observed in the positive-ion ESI-MS [ESI(+)-MS] spectra. Adducts with additional olefin units (up to three) were also observed. The ROM of the first cycloalkene unit was much easier than the subsequent additions. This finding was attributed to the $\pi$-complexation of the penultimate double bond to the ruthenium centre.

In another work with the CTL $\mathbf{4 3}$, the same authors evaluated the effect of the carbene substituent and the isotope effect on the metathesis rate. ${ }^{\mathbf{8 1}}$ Initially, the electron-sprayed intermediate, 43, was reacted with substituted styrenes, generating 47 (Scheme 22). The reaction rate of 47 with propene and norbornene was measured. A modest acceleration of the acyclic metathesis reaction was observed in the presence of the electron-withdrawing groups on the aryl group of the substituted ruthenium benzylidene. Moreover, the carbene complex formed from 47 and styrene- $\mathrm{d}_{8}$ exhibited secondary deuterium kinetic isotope effects for the acyclic metathesis of 1-butene and the ROM of norbornene in the formation of 44 and 48, respectively.

Remarkable findings, concerning the reversibility of ROM, were obtained with $\operatorname{ESI}(+)-M S$ experiments for bifunctional substrates, which offer the product of a ROM reaction the chance to undergo a non-degenerate RCM to form a complex that is isotopically distinguishable from the original complex in MS. The cyclopentane derivative does not react with $\mathbf{4 4}$ under the relatively mild conditions in this experiment; the cyclopentene derivative, instead, leads to approximately $10 \%$

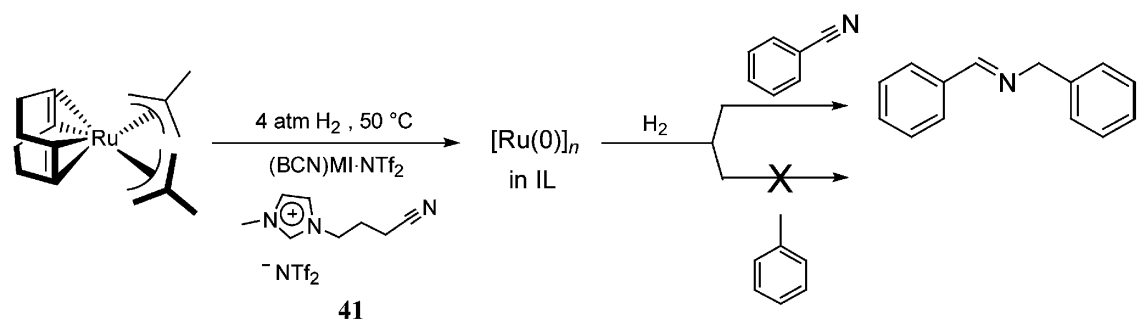

Scheme 19 Selective hydrogenation of benzonitrile, catalysed by $[R u(0)]_{n}$ NPs in a nitrile-functionalised IL. ${ }^{54}$ 

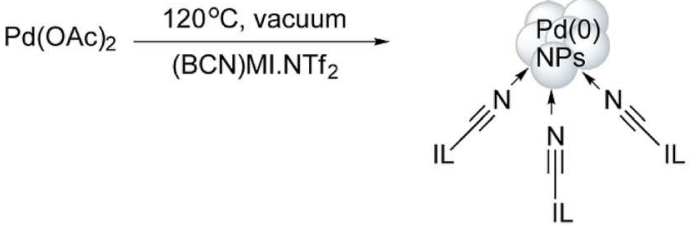

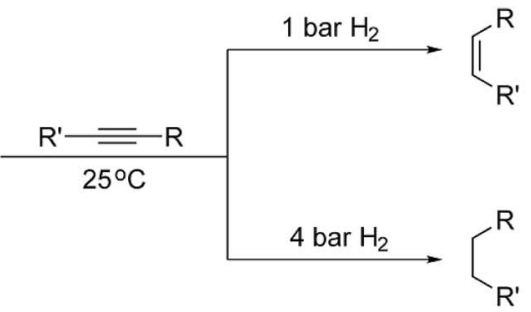

$\mathrm{R}=\mathrm{H}$, aryl, alkyl

Scheme 20 Hydrogenation of alkynes, which is controlled by a variable hydrogen gas pressure. ${ }^{60}$

conversion of 44 to 44-d6 (Scheme 23), indicating that the ROM of cyclopentene had occurred.

The same charge-tagged intermediate, 42 , was also used to study RCM through ESI(+)-MS experiments. ${ }^{82}$ Again, 43 was generated and, after a gas-phase reaction with ethylene, a charged, 14-electron, methylene ruthenium species was detected. Concerning the reactivity of $\mathrm{N}(\mathrm{Boc})(\text { allyl })_{2}$ and other diallyl compounds in RCM, it could be confirmed that all the monomolecular steps in the catalytic cycle of the RCM, following olefin coordination, are fast, and no intermediate can be accumulated to become detectable by ESI-MS. Additionally, alkylidene trialkylphosphane and phosphonium salts were easily detected and attributed to degradation products.

Cross-coupling reactions. The actual mechanism of the copper-catalysed coupling between phenols/amines and aryl/ vinyl halides (Ullmann coupling) is still under discussion. It

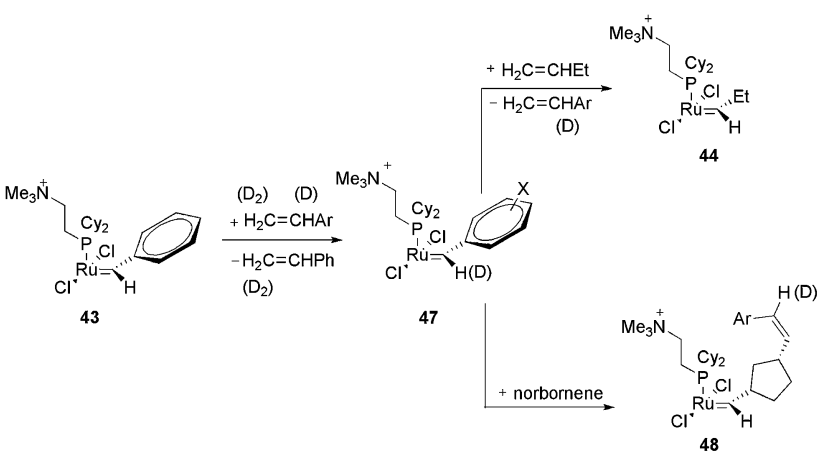

Scheme 22 Reaction of the electron-sprayed intermediate 43 with substituted styrenes, generating 47 and subsequently 44 and 48 .

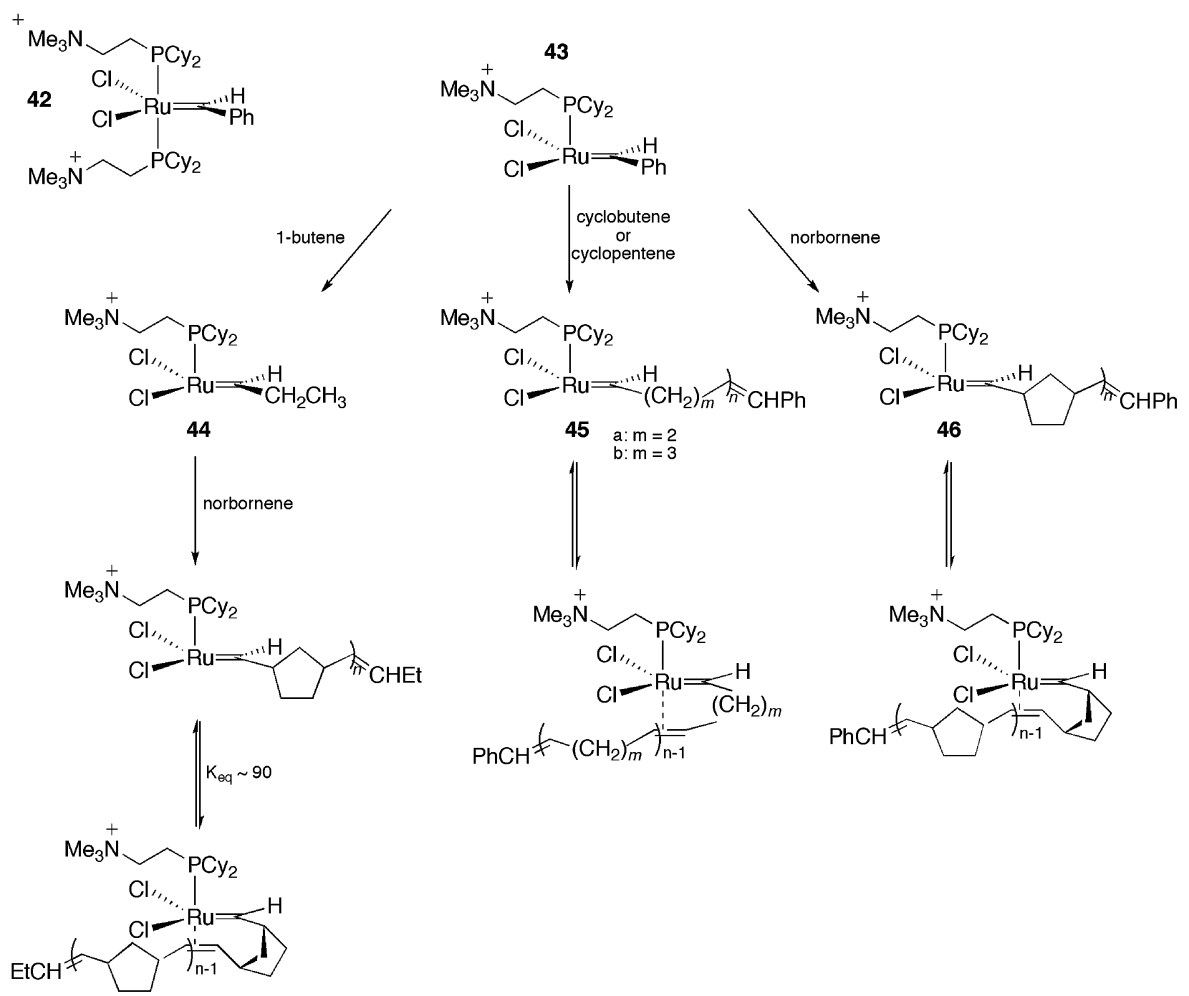

Scheme 21 CTLs in the ESI-MS detection of olefin metathesis intermediates. 

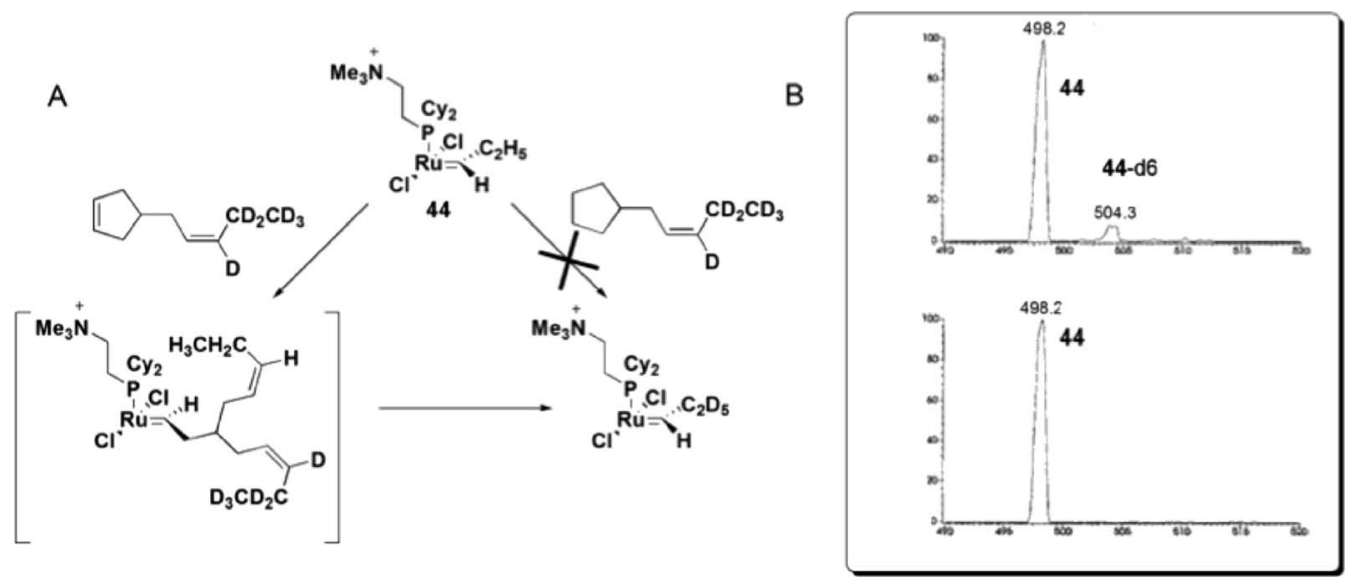

Scheme 23 (A) Reaction of 44 with 4-[(Z)-2-pentenyl-3,4,4,5,5,5-d6]-cyclopentene or 4-[(Z)-2-pentenyl-3,4,4,5,5,5-d6]-cyclopentane. (B) Top: ESI(+)-MS spectra of the reaction of 44 with a cyclopentene derivative, with the detection of 44-d6. Bottom: ESI(+)-MS spectra of the reaction of 44 with a cyclopentane derivative, with no detection of 44-d6. Adapted from ref. 81. Copyright (2000) American Chemical Society.

remains unclear whether carbon-halide bond activation occurs through an oxidative addition, $\mathrm{Cu}(\mathrm{I})-\mathrm{Cu}(\mathrm{III})$, process (as demonstrated in theoretical studies, ${ }^{83,84}$ reactions with welldefined $\mathrm{Cu}(\mathrm{III})$ compounds $^{85-87}$ and radical-detection tests ${ }^{88,89}$ ) or via a radical SET/IAT Cu(I)-Cu(II) pathway (as shown in theoretical studies $^{90}$ and ESI-MS experiments combined with radical-scavenger tests ${ }^{91}$ ). Another point of discussion is the putative formation of a nucleophilic copper species, preceding the organic-halide activation.

We recently described the synthesis and use of the phenanthroline-based ligands 49 and 50 in ESI(+)-MS experiments..$^{92}$ These cationic ligands were used as probes to detect the intermediates in the copper-catalysed coupling of $(E)$-bromostilbene with phenols (Scheme 24).

In this way, we were able to detect various intermediates (Fig. 4). Initially, intermediates 49a and 50a, corresponding to 49 and 50 coordinated to copper iodide, were observed. After the addition of phenol, base and vinyl bromide, various restingstate intermediates could be detected. Intermediates $49 \mathbf{b}$, in which a benzylic cleavage of the ligands had occurred, were similar to those described as very active catalysts in the arylation of phenols, even at very low concentrations of copper (10 ppm). ${ }^{93}$ Another important intermediate was 49c. In this

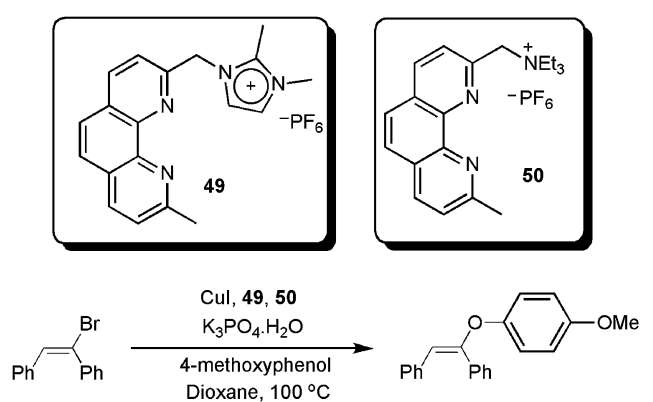

Scheme 24 CTLs 49 and 50 as probes to detect intermediates in the copper-catalysed Ullmann vinylation. species, the ionophilic probe was not cleaved and it contained a $\mathrm{Cu}-\mathrm{OAr}$ bond. The formation of $\mathrm{Cu}$-nucleophile species has been described in the arylation of phenols ${ }^{94}$ and for other nucleophiles. ${ }^{84,89,95-98}$ These species have been reported to be capable of reacting with aryl halides under mild conditions. $^{84,89,94-98}$ Moreover, $49 \mathrm{c}$ also indicates that the phenol moiety can act as a nucleophile as well as a ligand.

Based on the results obtained from ESI(+)-MS, preliminary kinetic studies and the radical-scavenger experiment, we could propose viable mechanistic pathways for the copper/1,10-phenanthroline-catalysed vinylation of phenols. At first, the coordination of the ligand to CuI takes place, providing a (phen)CuI species, which was detected as 49a and 50a in the ESI(+)-MS. Thereafter, an intermediate $\left[(\mathrm{phen})_{2} \mathrm{Cu}\right]^{+}$is formed. The formation of this intermediate was supported by the detection of $49 \mathrm{~b}$. Next, the $\mathrm{Cu}-\mathrm{OAr}$ intermediate was detected as $49 \mathrm{c}$, which is reported to be able to react with the organic halides. ${ }^{94}$ In terms of vinyl halide activation, as the radical pathway is not operational, we could argue in favour of an oxidative addition to form a $\mathrm{Cu}(\mathrm{III})$ intermediate. Although these species are not common, arylcopper(III) complexes have recently been identified, ${ }^{85,99,100}$ and arylcopper(III) intermediates containing nitrogen ligands are calculated to be kinetically accessible under the reaction conditions. ${ }^{89}$

Then, the [(phen)-CuOAr] intermediate would be reformed by a putative reductive elimination, followed by the attack of the phenoxide to form the copper species (Scheme 25).
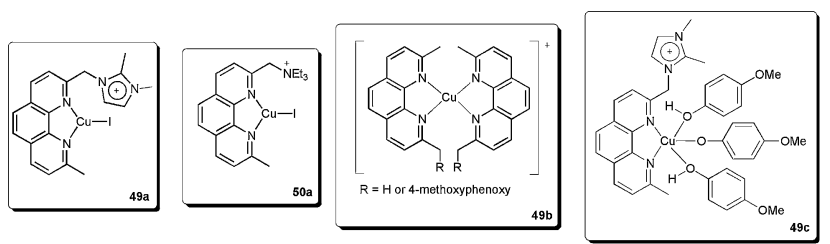

Fig. 4 Intermediates detected in ESI(+)-MS. 
The charge-tagged carboxylate ligand $\mathbf{5 1}$ was synthesised by Neto and co-workers. ${ }^{17}$ This ligand was reacted with $\mathrm{Cu}(\mathrm{OAc})_{2}$, $\mathrm{Ni}(\mathrm{OAc})_{2}$ and $\mathrm{Pd}(\mathrm{OAc})_{2}$, generating the corresponding monoand dicationic charged-tagged metal complexes 51a-51f (Scheme 26).

The ion-tagged complexes were characterised by ESI(+)-MS and $\operatorname{ESI}(+)-\mathrm{MS} / \mathrm{MS}$. For both the $\mathrm{Cu}$ and $\mathrm{Ni}$ complexes, the formation of metallic NHC carbenes was observed in the mass spectra. The palladium dicationic carboxylate was successfully used in the Heck and Suzuki reactions. In both reactions, the activity of $\mathbf{5 1 f}$ was greater than that of $\mathrm{Pd}(\mathrm{OAc})_{2}$. Although the palladium carbene was not observed in the ESI-MS experiments, the superior activity of the CTL was attributed to formation of this type of intermediate.

It must be mentioned that the same research group described the detection of this NHC carbene through negativeion ESI-MS [ESI(-)-MS] experiments of $\mathrm{COOH}^{-}$and $\mathrm{SO}_{3} \mathrm{H}$ substituted IL. In the gas phase, the doubly deprotonated (imidazolium and $\mathrm{COOH}$ or $\mathrm{SO}_{3} \mathrm{H}$ ) NHC carbene was detected. Moreover, the behaviour of these carbenes in the gas phase was similar to those in the liquid phase, because they can react with $\mathrm{CO}_{2}$ and generate organic carboxylates at the $\mathrm{C} 2$ positions of imidazolium. ${ }^{101,102}$

Key reaction intermediates associated with $\mathrm{Pd}$ catalysis could also be detected and characterized due to the presence of the charge tag on the Pd-complex (Scheme 27). ${ }^{103}$ The new charge-tagged Pd-complex is also shown to function as an active catalyst "on water" with the advantage of using cheaper and less reactive aryl chloride substrates in a phosphine-free version of the Heck reaction.

The use of tagged substrates ${ }^{19}$ and ligands ${ }^{104}$ has been described for the study of the intermediates of the palladiumcatalysed Sonogashira cross-coupling reaction through ESI(+)-

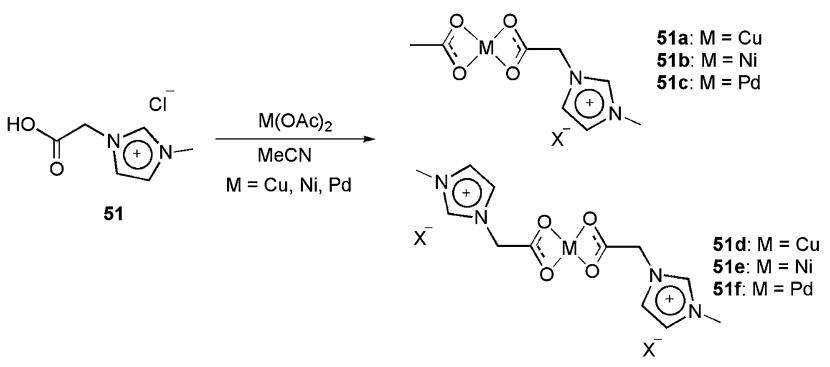

Scheme 26 Reaction of the charge-tagged carboxylate ligand 51 with $\mathrm{Cu}(\mathrm{OAc})_{2}, \mathrm{Ni}(\mathrm{Oac})_{2}$ and $\mathrm{Pd}(\mathrm{OAc})_{2}$, generating the corresponding mono- and dicationic charge-tagged metal complexes.

MS and ESI(-)-MS experiments. The commercial salt of monosulfonated triphenylphosphine $[\mathrm{Na}]^{+}\left[\mathrm{PPh}_{2}\left(m-\mathrm{C}_{6} \mathrm{H}_{4} \mathrm{SO}_{3}\right)\right]^{-}$ $\left([\mathrm{Na}]^{+}[52]^{-}\right)$was used as a precursor to obtain $\mathrm{CH}_{2} \mathrm{Cl}_{2}$-soluble $\left[\left(\mathrm{Ph}_{3} \mathrm{P}\right)_{2} \mathrm{~N}\right]^{+}\left[\mathrm{PPh}_{2}\left(m-\mathrm{C}_{6} \mathrm{H}_{4} \mathrm{SO}_{3}\right)\right]^{-}\left([\mathrm{PPN}]^{+}[\mathbf{5 2}]^{-}\right)$. An equimolar amount of the CTL $[\mathrm{PPN}][52]$ was added to a $\mathrm{Pd}\left(\mathrm{PPh}_{3}\right)_{4}$ solution. This mixture provided an ESI(-)-MS spectrum that consisted of only $\left[\mathrm{Pd}\left(5^{-}\right) \mathrm{PPh}_{3}\right]$ and $\left[\mathrm{Pd}\left(5^{-}\right)\left(\mathrm{PPh}_{3}\right)_{2}\right]$. After PhI was added, all of the $\operatorname{Pd}(0)$ species were consumed and the oxidative addition product $\left[\mathrm{Pd}\left(\mathbf{5 2}^{-}\right)\left(\mathrm{PPh}_{3}\right)(\mathrm{Ph})(\mathrm{I})\right]$ could be detected. Upon the addition of phenylacetylene and $\mathrm{NEt}_{3}$, the intermediate $\left[\mathrm{Pd}\left(\mathbf{5 2}^{-}\right)\left(\mathrm{PPh}_{3}\right)(\mathrm{Ph})\left(\mathrm{C}_{2} \mathrm{Ph}\right)\right]$ was formed (Scheme 28A). Additionally, by using piperidine, the intermediate $\left[\mathrm{Pd}\left(5^{-}\right)\left(\mathrm{HNR}_{2}\right)(\mathrm{Ph})(\mathrm{I})\left(\mathrm{HC}_{2} \mathrm{Ph}\right)\right](\mathrm{m} / \mathrm{z}=838$; Scheme $28 \mathrm{~B})$ could be identified. Collision-induced dissociation (CID) experiments were performed on this complex and the loss of phenylacetylene and then piperidine was observed. Moreover, there was no detected loss of benzene, indicating that the triple bond is $\pi$ coordinated in this complex. These results led to the catalytic cycle proposed in scheme 28C.

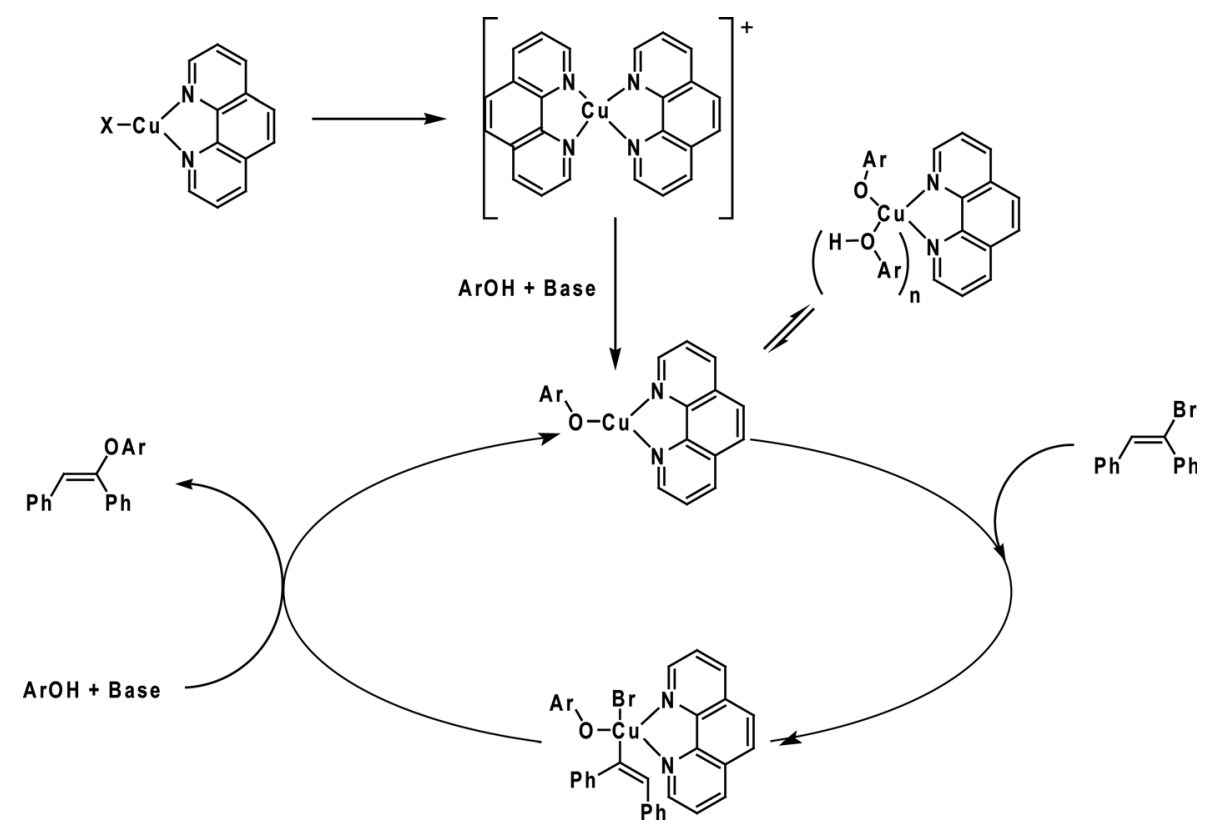

Scheme 25 Catalytic cycle proposed for the copper-catalysed vinylation of phenols. Adapted from ref. 92. 


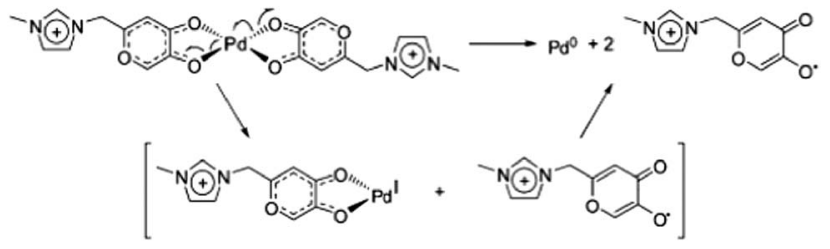

Scheme 27 Proposed Pd-reduction by ligand decomposition.

Olefin hydrogenation. Scheme 29 has been studied in detail, ${ }^{\mathbf{1 0 5}-108}$ and both the in-cycle (B-E) and off-cycle (A, F-J) species have been characterised. For this reason, the reaction can be used to evaluate the reliability of ESI-MS for detecting intermediates during catalysis. However, because all of the species are neutral, it is necessary to charge-tag them.

Therefore, $\mathrm{Ph}_{2} \mathrm{P}\left(\mathrm{CH}_{2}\right)_{n} \mathrm{PPh}_{2}(n=1,2,4,6)$ diphosphines were selectively monobenzylated to produce charge-tagged phosphonium/phosphine $\left[\mathrm{Ph}_{2} \mathrm{P}\left(\mathrm{CH}_{2}\right)_{n} \mathrm{PPh}_{2} \mathrm{CH}_{2} \mathrm{Ph}\right]^{+}$ligands. These CTLs were used to dope $\mathrm{RhCl}\left(\mathrm{PPh}_{3}\right)_{3}$ under hydrogenation conditions in order to detect the reaction intermediates in ESI(+)-MS. ${ }^{109}$

Ligand $53(n=4)$ was added to a solution of $\operatorname{RhCl}\left(\mathrm{PPh}_{3}\right)_{3}$ in chlorobenzene. The reaction was analysed before $\mathrm{H}_{2}$ was added, after $\mathrm{H}_{2}$ was added and after the addition of cyclohexene. The results are summarised in Table 1 . Initially, various intermediates, in which either one or two $\mathrm{PPh}_{3}$ ligands had been replaced by 53, were detected (for instance $\left[\mathrm{RhCl}\left(\mathrm{PPh}_{3}\right)\left(\mathbf{5 3}^{+}\right)_{2}\right]$ and $\left.\left[\mathrm{RhCl}\left(\mathrm{PPh}_{3}\right)_{2}\left(\mathbf{5 3}^{+}\right)\right]\right)$. The reactive B-type (see the catalytic cycle) species $\left[\operatorname{RhCl}\left(\mathrm{PPh}_{3}\right)\left(\mathbf{5 3}^{+}\right)\right]$and $\left[\operatorname{RhCl}\left(\mathbf{5 3}^{+}\right)_{2}\right]_{2}$ were also detected; however, by varying the cone voltage it could be demonstrated that these species were formed by the fragmentation of the A-type species. When hydrogen was bubbled through the solution, an immediate change in the mass spectrum was observed. Even at a low cone voltage, a significant amount of the three-coordinate species $\left[\mathrm{RhCl}\left(\mathrm{PPh}_{3}\right)\left(\mathbf{5 3}^{+}\right)\right]$appeared. In addition, the hydrogenated species $\left[\mathrm{RhCl}\left(\mathrm{PPh}_{3}\right)\left(\mathbf{5 3}^{+}\right)_{2} \mathrm{H}_{2}\right], \quad\left[\mathrm{RhCl}\left(\mathrm{PPh}_{3}\right)_{2}\left(\mathbf{5 3}^{+}\right) \mathrm{H}_{2}\right]$, $\left[\mathrm{RhCl}\left(\mathrm{PPh}_{3}\right)\left(\mathbf{5 3}^{+}\right)_{2} \mathrm{H}_{2} \mathrm{BF}_{4}\right],\left[\mathrm{Rh}_{2} \mathrm{Cl}_{2}\left(\mathrm{PPh}_{3}\right)_{3}\left(\mathbf{5 3}^{+}\right) \mathrm{H}_{2}\right]$ and $\left[\mathrm{Rh}_{2} \mathrm{Cl}_{2}(-\right.$ $\left.\mathrm{PPh}_{3}\right)_{3}\left(5^{+}\right) \mathrm{H}_{4}$ ] could be detected. After the addition of cyclohexene, despite the change in speciation, no alkene-coordinated (D-type) species were detected. However, bubbling ethylene through the hydrogen-saturated solution resulted in the

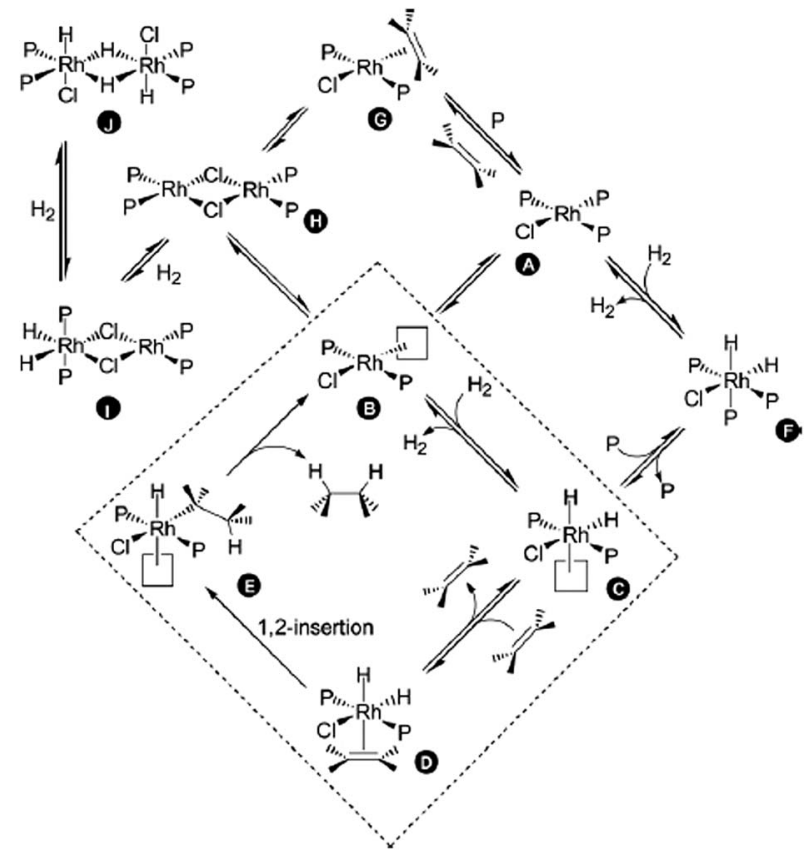

Scheme 29 Catalytic cycle of the RhCl(PPh$)_{3}$-catalysed hydrogenation of olefins. Reproduced from ref. 109 with permission from the Royal Society of Chemistry.

appearance of a small amount (ca. $2 \%)$ of $\left[\mathrm{RhCl}\left(\mathrm{PPh}_{3}\right)\left(\mathbf{5 3}^{+}\right)\left(\mathrm{H}_{2}-\right.\right.$ $\left.\left.\mathrm{C}=\mathrm{CH}_{2}\right)\right]$ (G-type). In summary, by using this method, all the offcycle (A, F-I) species could be identified rapidly.

An iron(III) complex with three ionic tags, 54, was applied to the reduction of olefins in imidazolium-based ILs under oxidative conditions. ${ }^{\mathbf{1 1 0}}$ The catalyst was very efficient in promoting reactions involving biomass derivatives. ICP analysis revealed that the use of this catalyst greatly prevented catalyst leaching, and as little as $2 \mathrm{ppm}$ of Fe was detected in the organic phase of the reaction. This indicates the efficient anchorage of the complex in the $\mathrm{BMI} \cdot \mathrm{NTf}_{2} \mathrm{IL}$. Moreover, the activity of this complex remained unchanged after ten runs. Some important mechanistic insights for this new reaction were also provided, mostly based on ESI quadrupole time-of-flight MS (ESI-QTOFMS), as can be seen in Scheme 30. Based on these experiments,
A)

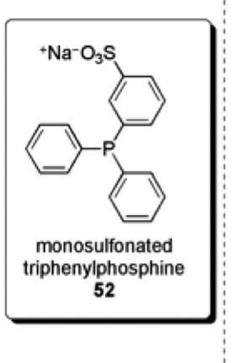

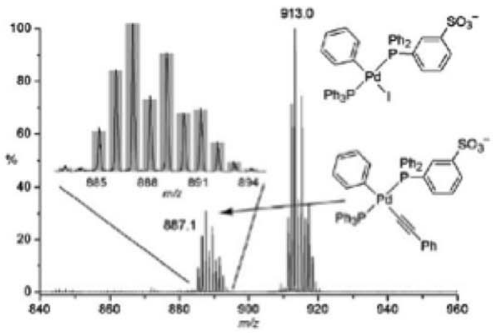

C)

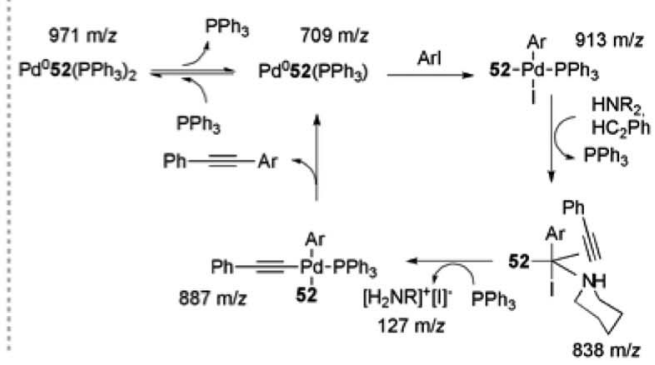

Scheme 28 (A) Structure of monosulfonated triphenylphosphine (52). (B) ESI(-)-MS of Pd(PPh $)_{4}+[P P N]\left[52^{-}\right]+100 \times\left(\mathrm{Phl}^{-}+\mathrm{PhC}_{2} \mathrm{H}+\mathrm{NEt} 3\right)$ in $\mathrm{CH}_{2} \mathrm{Cl}_{2}$. Inset: isotope pattern match for $\left[\mathrm{Pd}\left(52^{-}\right)\left(\mathrm{PPh}_{3}\right)(\mathrm{Ph})\left(\mathrm{C}_{2} \mathrm{Ph}\right)\right]$. (C) Intermediates that were directly observed by ESI(-)-MS and the proposed catalytic cycle. Reproduced from ref. 104 with permission from the Royal Society of Chemistry. 
Table 1 Species detected in ESI(+)-MS for the reaction of $\mathrm{RhCl}\left(\mathrm{PPh}_{3}\right)_{3}$ with 53. Adapted from ref. 109

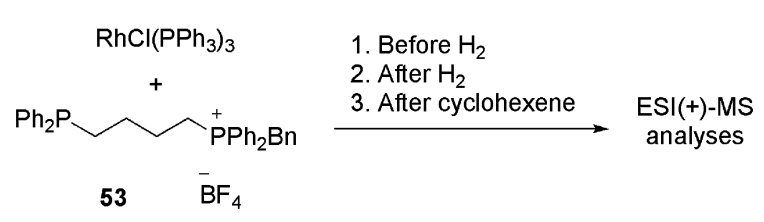

\begin{tabular}{|c|c|c|c|c|c|c|}
\hline Species & Cycle & Identity & $m / z$ & $\begin{array}{l}\text { Intensity before } \\
\mathrm{H}_{2} \text { addition }\end{array}$ & $\begin{array}{l}\text { Intensity after } \\
\mathrm{H}_{2} \text { addition }\end{array}$ & $\begin{array}{l}\text { Intensity after cyclohexene } \\
\text { addition }\end{array}$ \\
\hline Free ligand & & $53^{+}$ & 517.2 & 13 & 13 & 4 \\
\hline & B & {$\left[\mathrm{RhCl}\left(\mathrm{PPh}_{3}\right)\left(53^{+}\right)\right]$} & 917.2 & 24 & 100 & 6 \\
\hline $\mathrm{RhP}_{3}^{+}$ & & {$\left[\mathrm{Rh}\left(\mathrm{PPh}_{3}\right)_{3}\right]^{+}$} & 889.2 & 15 & 8 & 30 \\
\hline \multirow[t]{4}{*}{$\mathrm{RhClP}_{3}$} & A & {$\left[\operatorname{RhCl}\left(\mathrm{PPh}_{3}\right)\left(\mathbf{5 3}^{+}\right)_{2}\right]$} & 717.2 & 16 & 9 & $<1$ \\
\hline & $\mathrm{F}$ & {$\left[\mathrm{RhCl}\left(\mathrm{PPh}_{3}\right)_{2}\left(\mathbf{5 3}^{+}\right) \mathrm{H}_{2}\right]$} & 1181.3 & - & 77 & $<1$ \\
\hline & A & {$\left[\operatorname{RhCl}\left(\mathrm{PPh}_{3}\right)\left(\mathbf{5 3}^{+}\right)_{2} \mathrm{BF}_{4}\right]$} & 1521.4 & 4 & $<1$ & 2 \\
\hline & $\mathrm{F}$ & {$\left[\mathrm{RhCl}\left(\mathrm{PPh}_{3}\right)\left(\mathbf{5 3}^{+}\right)_{2} \mathrm{H}_{2} \mathrm{BF}_{4}\right]$} & 1523.4 & - & 2 & $<1$ \\
\hline \multirow[t]{3}{*}{$\mathrm{Rh}_{2} \mathrm{Cl}_{2} \mathrm{P}_{4}$} & $\mathrm{H}$ & {$\left[\mathrm{Rh}_{2} \mathrm{Cl}_{2}\left(\mathrm{PPh}_{3}\right)_{3}\left(\mathbf{5 3}^{+}\right)\right]$} & 1581.2 & 6 & 2 & 3 \\
\hline & I & {$\left[\mathrm{Rh}_{2} \mathrm{Cl}_{2}\left(\mathrm{PPh}_{3}\right)_{3}\left(\mathbf{5 3}^{+}\right) \mathrm{H}_{2}\right]$} & 1583.2 & - & 2 & $<1$ \\
\hline & $\mathrm{J}$ & {$\left[\mathrm{Rh}_{2} \mathrm{Cl}_{2}\left(\mathrm{PPh}_{3}\right)_{3}\left(53^{+}\right) \mathrm{H}_{4}\right]$} & 1585.2 & - & $<1$ & $<1$ \\
\hline
\end{tabular}

a catalytic cycle that includes a high-valence Fe atom was proposed.

Metal-catalysed multicomponent Biginelli reactions. The Biginelli reaction $^{\mathbf{1 1 1}}$ is an acid-catalyzed, three-component reaction between an aldehyde, a $\beta$-ketoester and urea that allows the rapid and facile synthesis of dihydropyrimidones, which are interesting compounds with potential for pharmaceutical applications.

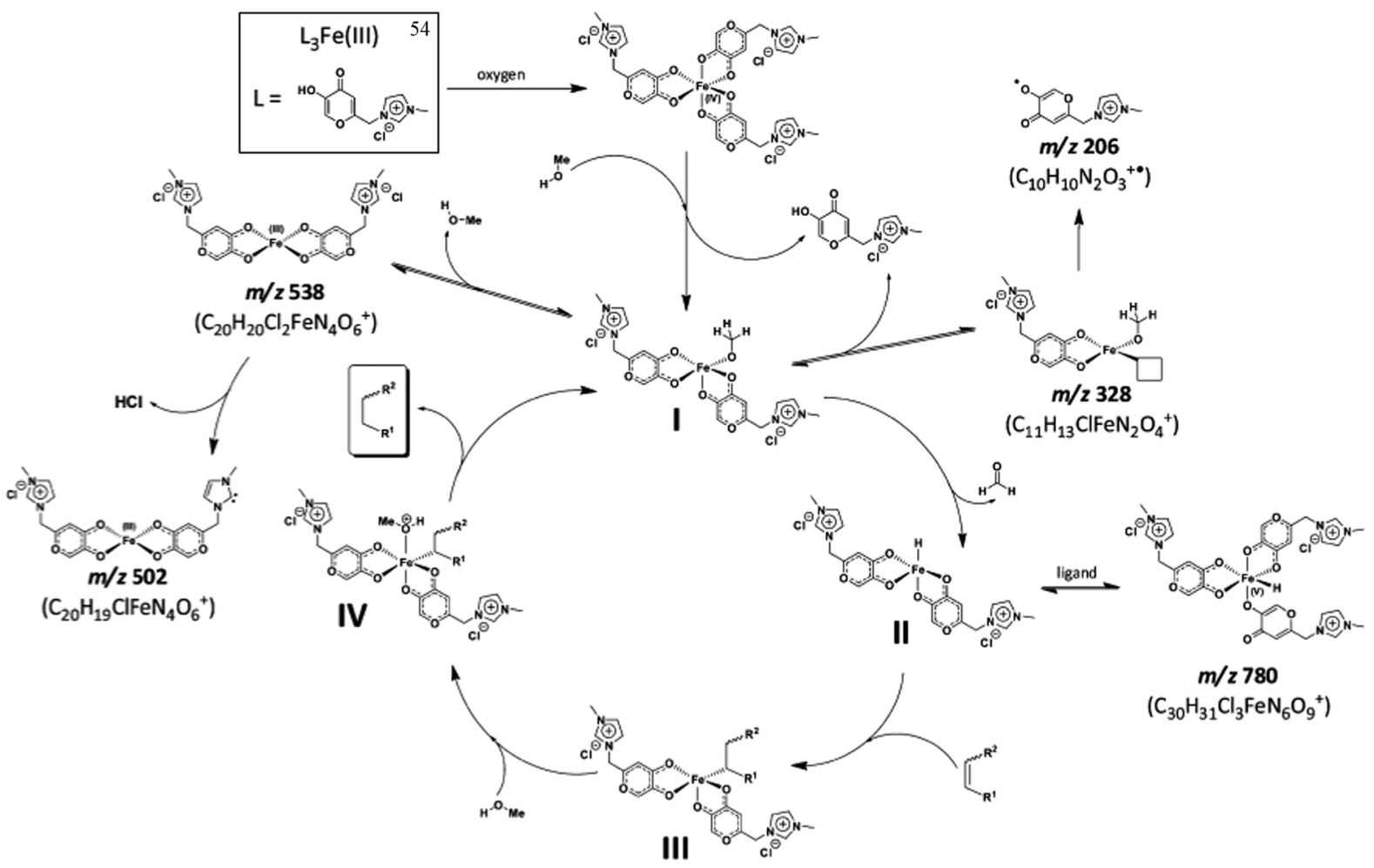

Scheme 30 Proposed catalytic cycle for olefin reduction catalysed by 54 in the presence of methanol. Major ions detected in the ESI(+)-QTOFMS experiments are indicated by their $\mathrm{m} / \mathrm{z}$ values, and proposals for the origins of the intermediates are shown. Reprinted with permission from ref. 112. Copyright (2012) American Chemical Society. 
This reaction catalysed by a Lewis acid in an IL was evaluated by Neto and co-workers using ESI-MS. ${ }^{112}$ This multi-component reaction was used to obtain the biologically active 3,4-dihydropyrimidin-2(1H)-one (DHPMs). As indicated by ESI-MS experiments, the ionic medium plays a fundamental role in this synthesis, owing to the stabilisation of the charged intermediates. Presently, there are three major pathways accepted for this reaction: (i) the iminium, (ii) the Knoevenagel and (iii) the enamine mechanisms. ${ }^{113-115}$ Through this work, the authors could detect some key intermediates and products of the reaction by using ESI(+)-MS(/MS) (Fig. 5). The online monitoring of the Biginelli reaction allowed the authors to detect and characterise the so-called iminium intermediate (Fig. 5E), indicating that, under these conditions, the preferred mechanistic pathway is the iminium mechanism. No key intermediate of the enamine mechanism could be detected.

This reaction has also been studied using the new ion-tagged iron catalysts 55 and 56 (Fig. 6) in ILs. ${ }^{116}$ High yields were obtained and the system could be recovered and reused up to eight times. High-resolution ESI-QTOF-MS experiments were performed to determine the reaction mechanism.

The three proposed mechanisms for the Biginelli reaction were investigated by ESI-QTOF-MS and MS/MS. Only the intermediates from the iminium mechanism were detected. No intermediates from the Knoevenagel or enamine pathways were found.

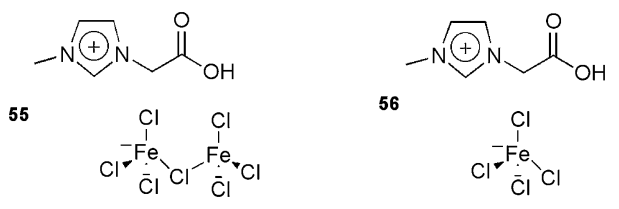

Fig. 6 lon-tagged iron catalysts used in the Biginelli reaction.

Iron-catalysed olefin epoxidation. The ionically tagged imidazolium-based iron(III) complex 54 was used in the oxidation of alkenes in imidazolium-based ILs in air (also hydrogen peroxide). ${ }^{48}$ The epoxidised olefin was obtained in very good yields (84-91\%) and at least ten recycling reactions could be performed. ESI-QTOF-MS experiments were performed to achieve a better understanding of the active catalytic species that promoted epoxidation. The results pointed firmly towards two different oxidation states of the metal centre [Fe(Iv) and $\mathrm{Fe}(\mathrm{v})]$, depending mostly on the selection of the oxidising agent. Hydrogen peroxide oxidation may proceed through a radical pathway and may use a Fe(Iv) species in its catalytically active form, whereas air oxidation may favour a concerted mechanism with an $\mathrm{Fe}(\mathrm{v})$-based intermediate as the active species to promote epoxidation. Some important mechanistic insights could be provided based on the ESI-QTOF-MS results for the oxidation reaction, which clearly indicated that oxidation can
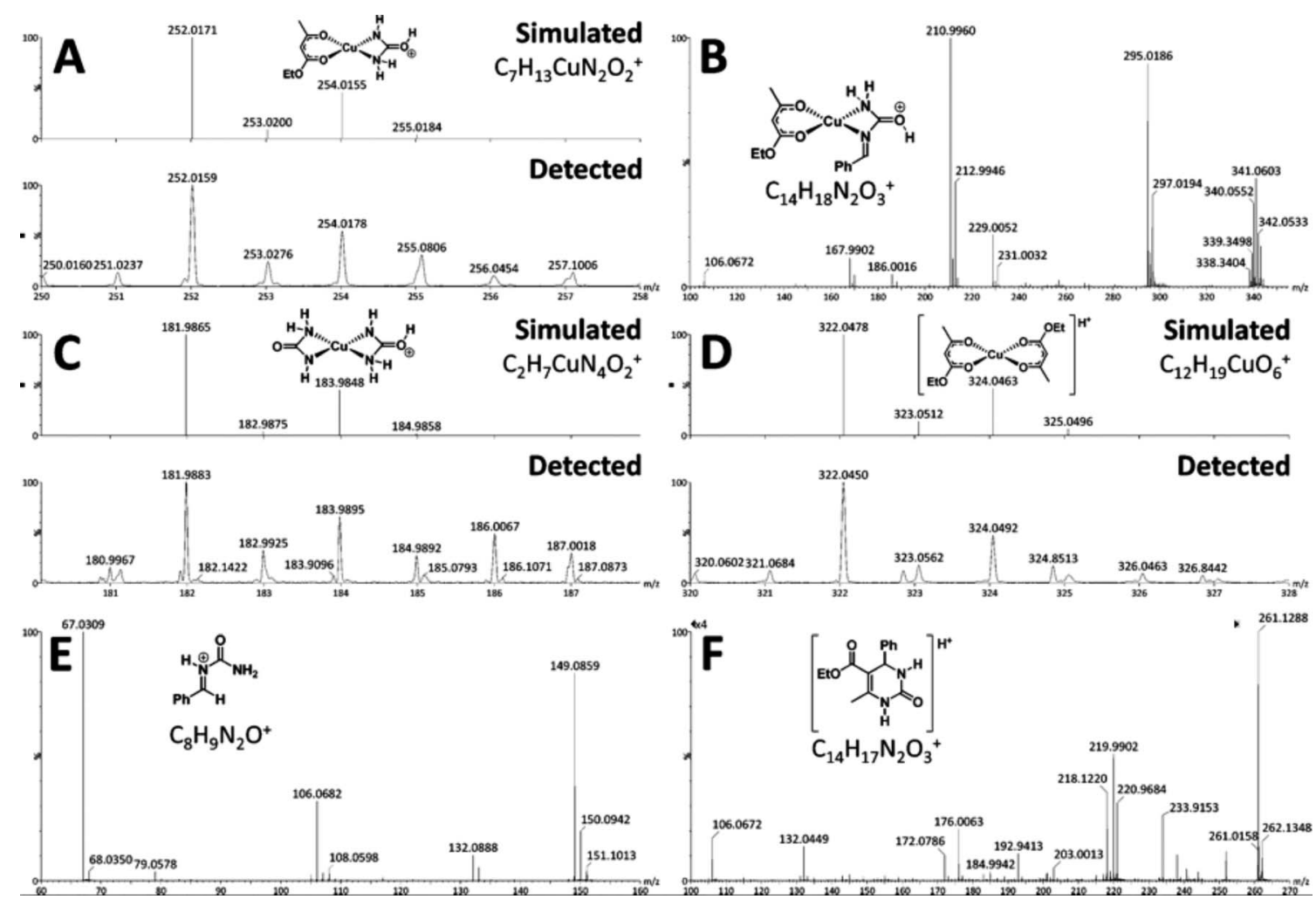

Fig. 5 Key intermediates and products detected (and characterised) by ESI(+)-MS: (A) $\mathrm{C}_{7} \mathrm{H}_{13} \mathrm{CuN}_{2} \mathrm{O}_{2}{ }^{+}$; (B) $\mathrm{C}_{14} \mathrm{H}_{18} \mathrm{~N}_{2} \mathrm{O}_{3}{ }^{+}$; (C) $\mathrm{C}_{2} \mathrm{H}_{7} \mathrm{CuN} \mathrm{O}_{4} \mathrm{O}_{2}^{+}$; (D) $\mathrm{C}_{12} \mathrm{H}_{19} \mathrm{CuO}_{6}{ }^{+}$; (E) iminium intermediate $\mathrm{C}_{8} \mathrm{H}_{9} \mathrm{~N}_{2} \mathrm{O}^{+}$; and (F) $\mathrm{C}_{14} \mathrm{H}_{17} \mathrm{~N}_{2} \mathrm{O}_{3}{ }^{+}$. Reprinted with permission from ref. 112. Copyright (2012) American Chemical Society. 
take place by two different pathways, depending on the reaction conditions, that is, a radical or a concerted mechanism.

\section{Conclusions and perspectives}

It is evident that, with the advent of IL-phase chemistry, a new class of organic charged ligands has emerged. Contrary to classical water-phase catalysis, for which negatively charged ligands (in most cases, P-containing ligands) are used, IL-phase catalysis is mainly based on positively charged ligands. This is necessary, because an incorrect solvent pair can lead to low conversions, poor product extraction, no phase separation and catalyst lixiviation. Using this approach, various classical homogeneous catalytic processes have been successfully transposed to liquid-liquid biphasic catalysis. However, so far, it is only in rare cases that the changes to the mechanism and selectivity of the transformations, imposed by the intrinsic ionic nature of the media, have been addressed. Moreover, even fewer insights have been gathered for the supramolecular organisation imposed by these highly organised fluids on the catalytically active sites. Therefore, a more detailed investigation on the mechanistic pathway in ionic media is necessary to allow the design of more efficient catalysts (for improved conversion, selectivity and sustainable catalytic processes). Notably, CTLs have also been used with a high degree of success in colloidal catalytic process.

It also appears that the use of CTLs has opened a new window of opportunities for the investigation of reaction pathways through ESI-MS. This approach has already proven to be one of the most important tools for detecting several species involved in various catalytic reactions directly from the solution. It is also expected that, in view of the virtually non-volatile nature of ILs, other techniques will be used to probe and monitor in situ reactions performed in solution, such as techniques requiring a high vacuum and those that are currently limited to solid-state chemistry, for example, X-ray photoelectron spectroscopy, low-energy ion scattering spectroscopy and TEM. This may also lead to important in situ solution-phase information that can be used for the design of new and more efficient catalysts.

\section{Acknowledgements}

We acknowledge the following Brazilian agencies for financial support: CNPq, FAPERGS, CAPES, INCT-Catal. and MCTI.

\section{References}

1 M. H. Abraham, P. L. Grellier, J.-L. M. Abboud, R. M. Doherty and R. W. Taft, Can. J. Chem., 1988, 66, 2673-2686.

2 C. C. Tzschucke, C. Markert, W. Bannwarth, S. Roller, A. Hebel and R. Haag, Angew. Chem., Int. Ed., 2002, 41, 3964-4000.

3 J.-I. Yoshida and K. Itami, Chem. Rev., 2002, 102, 3693-3716.

4 E. de Wolf, G. van Koten and B.-J. Deelman, Chem. Soc. Rev., 1999, 28, 37-41.
5 J.-M. Vicent, Top. Curr. Chem., 2012, 308, 153-174.

6 A. Köllhofer and H. Plenio, Chem.-Eur. J., 2003, 9, 14161425.

7 M. an der Heiden and H. Plenio, Chem.-Eur. J., 2004, 10, 1789-1797.

8 J. R. Gregório, R. Rosa, A. N. F. Mendes and J. C. Bayón, Catal. Lett., 2011, 141, 977-981.

9 F. Joó, Acc. Chem. Res., 2002, 35, 738-745.

10 B. Cornils, Org. Process Res. Dev., 1998, 2, 121-127.

11 B. Cornils, J. Mol. Catal. A: Chem., 1999, 143, 1-10.

12 N. Pinault and D. W. Bruce, Coord. Chem. Rev., 2003, 241, 1-25.

13 B. Cornils and E. G. Kuntz, J. Organomet. Chem., 1995, 502, 177-186.

14 E. G. Kuntz, Chem. Tech., 1987, 17, 570-575.

15 E. G. Kuntz, Patent Fr. 2.314.910 1975.

16 R. Sebesta, I. Kmentova and S. Toma, Green Chem., 2008, 10, 484-496.

17 F. F. D. Oliveira, M. R. dos Santos, P. M. Lalli, E. M. Schmidt, P. Bakuzis, A. A. M. Lapis, A. L. Monteiro, M. N. Eberlin and B. A. D. Neto, J. Org. Chem., 2011, 76, 10140-10147.

18 C. S. Consorti, G. L. P. Aydos and J. Dupont, Chem. Commun., 2010, 46, 9058-9060.

19 K. L. Vikse, Z. Ahmadi, C. C. Manning, D. A. Harrington and J. S. McIndoe, Angew. Chem., Int. Ed., 2011, 50, 8304-8306.

20 C. Adlhart and P. Chen, Helv. Chim. Acta, 2000, 83, 21922196.

21 D. M. Chisholm and J. S. McIndoe, Dalton Trans., 2008, 30, 3933-3945.

22 M. Lombardo and C. Trombini, ChemCatChem, 2010, 2, 135-145.

23 C. S. Consorti, G. L. P. Aydos, G. Ebeling and J. Dupont, Organometallics, 2009, 28, 4527-4533.

24 C. S. Consorti, G. L. P. Aydos, G. Ebeling and J. Dupont, Org. Lett., 2008, 10, 237-240.

25 G. Liu and J. Wang, Angew. Chem., Int. Ed., 2010, 49, 44254429.

26 N. Clousier, A. Filippi, E. Borré, E. Guibal, C. Crévisy, F. Caijo, M. Mauduit, I. Dez and A.-C. Gaumont, ChemSusChem, 2014, 7, 1043-1045.

27 R. Wang, J. C. Xiao, B. Twamley and J. M. Shreeve, Org. Biomol. Chem., 2007, 5, 671-678.

28 J. C. Xiao, B. Twamley and J. M. Shreeve, Org. Lett., 2004, 6 , 3845-3847.

29 J.-C. Xiao and J. n. M. Shreeve, J. Org. Chem., 2005, 70, 30723078.

30 R. H. Wang, M. M. Piekarski and J. M. Shreeve, Org. Biomol. Chem., 2006, 4, 1878-1886.

31 M. Lombardo, M. Chiarucci and C. Trombini, Green Chem., 2009, 11, 574-579.

32 C. C. Cassol, A. P. Umpierre, G. Machado, S. I. Wolke and J. Dupont, J. Am. Chem. Soc., 2005, 127, 3298-3299.

33 C. J. Mathews, P. J. Smith, T. Welton, A. J. P. White and D. J. Williams, Organometallics, 2001, 20, 3848-3850.

34 L. Xu, W. Chen, J. Ross and J. Xiao, Org. Lett., 2001, 3, 295297. 
35 D. Zhao, Z. Fei, T. J. Geldbach, R. Scopelliti and P. J. Dyson, J. Am. Chem. Soc., 2004, 126, 15876-15882.

36 C. Chiappe, D. Pieraccini, D. Zhao, Z. Fei and P. J. Dyson, Adv. Synth. Catal., 2006, 348, 68-74.

37 C.-L. Xia, C.-F. Xie, Y.-F. Wu, H.-M. Sun, Q. Shen and Y. Zhang, Org. Biomol. Chem., 2013, 11, 8135-8144.

38 R. B. Bedford, D. W. Bruce, R. M. Frost, J. W. Goodby and M. Hird, Chem. Commun., 2004, 2822-2823.

39 G. Cahiez, V. Habiak, C. Duplais and A. Moyeux, Angew. Chem., Int. Ed., 2007, 46, 4364-4366.

40 H.-n. Deng, Y.-l. Xing, C.-l. Xia, H.-m. Sun, Q. Shen and Y. Zhang, Dalton Trans., 2012, 41, 11597-11607.

41 T. J. Geldbach and P. J. Dyson, J. Am. Chem. Soc., 2004, 126, 8114-8115.

42 R. Sebesta and F. Bilcik, Tetrahedron: Asymmetry, 2009, 20, 1892-1896.

43 M. J. Bravo, I. Favier, N. Saffon, R. M. Ceder, G. Muller, M. Gómez and M. Rocamora, Organometallics, 2014, 33, 771-779.

44 I. Favier, A. B. Castillo, C. Godard, S. Castillon, C. Claver, M. Gomez and E. Teuma, Chem. Commun., 2011, 47, 7869-7871.

45 X. D. Feng, B. Pugin, E. Kuesters, G. Sedelmeier and H. U. Blaser, Adv. Synth. Catal., 2007, 349, 1803-1807.

46 Y. W. Zhao, H. M. Huang, J. P. Shao and C. G. Xia, Tetrahedron: Asymmetry, 2011, 22, 769-774.

47 Z. M. Zhou, Z. H. Li, X. Y. Hao, J. Zhang, X. Dong, Y. Q. Liu, W. W. Sun, D. Cao and J. L. Wang, Org. Biomol. Chem., 2012, 10, 2113-2118.

48 M. R. dos Santos, J. R. Diniz, A. M. Arouca, A. F. Gomes, F. C. Gozzo, S. M. Tamborim, A. L. Parize, P. A. Z. Suarez and B. A. D. Neto, ChemSusChem, 2012, 5, 716-726.

49 W. Liu, Q. X. Wan and Y. Liu, Monatsh. Chem., 2010, 141, 859-865.

50 Y. Liu, H. J. Zhang, Y. Lu, Y. Q. Cai and X. L. Liu, Green Chem., 2007, 9, 1114-1119.

51 R. De Paula, M. M. Q. Simoes, M. Neves and J. A. S. Cavaleiro, Catal. Commun., 2008, 10, 57-60.

52 R. De Paula, M. M. Q. Simoes, M. Neves and J. A. S. Cavaleiro, J. Mol. Catal. A: Chem., 2011, 345, 1-11.

53 X. Jin, K. Zhao, F. Cui, F. Kong and Q. Liu, Green Chem., 2013, 15, 3236-3242.

54 J. D. Scholten, B. C. Leal and J. Dupont, ACS Catal., 2011, 2, 184-200.

55 R. R. Dykeman, N. Yan, R. Scopelliti and P. J. Dyson, Inorg. Chem., 2011, 50, 717-719.

56 B. Léger, A. Denicourt-Nowicki, H. l. n. Olivier-Bourbigou and A. Roucoux, Inorg. Chem., 2008, 47, 90909096.

57 B. Léger, A. Denicourt-Nowicki, H. Olivier-Bourbigou and A. Roucoux, Tetrahedron Lett., 2009, 50, 6531-6533.

58 M. H. G. Prechtl, J. D. Scholten and J. Dupont, J. Mol. Catal. A: Chem., 2009, 313, 74-78.

59 M. H. G. Prechtl, M. Scariot, J. D. Scholten, G. Machado, S. r. R. Teixeira and J. Dupont, Inorg. Chem., 2008, 47, 8995-9001.
60 R. Venkatesan, M. H. G. Prechtl, J. D. Scholten, R. P. Pezzi, G. Machado and J. Dupont, J. Mater. Chem., 2011, 21, 30303036.

61 L. S. Santos, Eur. J. Org. Chem., 2008, 2008, 235-253.

62 M. N. Eberlin, Eur. J. Mass Spectrom., 2007, 13, 19-28.

63 D. Schröder, Acc. Chem. Res., 2012, 45, 1521.

64 P. A. Enquist, P. Nilsson, P. Sjoberg and M. Larhed, J. Org. Chem., 2006, 71, 8779-8786.

65 C. Raminelli, M. H. G. Prechtl, L. S. Santos, M. N. Eberlin and J. V. Comasseto, Organometallics, 2004, 23, 39903996.

66 N. Taccardi, R. Paolillo, V. Gallo, P. Mastrorilli, C. F. Nobile, M. Raisanen and T. Repo, Eur. J. Inorg. Chem., 2007, 46454652.

67 D. Harakat, J. Muzart and J. Le Bras, RSC Adv., 2012, 2, 3094-3099.

68 C. Vicent, M. Viciano, E. Mas-Marza, M. Sanau and E. Peris, Organometallics, 2006, 25, 3713-3720.

69 A. B. Chaplin and P. J. Dyson, Organometallics, 2007, 26, 2447-2455.

70 A. T. Lubben, J. S. McIndoe and A. S. Weller, Organometallics, 2008, 27, 3303-3306.

71 X. S. He, S. S. Zhang, Y. L. Guo, H. Y. Wang and G. Q. Lin, Organometallics, 2012, 31, 2945-2948.

72 M. A. Schade, J. E. Feckenstem, P. Knochel and K. Koszinowski, J. Org. Chem., 2010, 75, 6848-6857.

73 L. Fiebig, H. G. Schmalz and M. Schafer, Int. J. Mass spectrom., 2011, 308, 307-310.

74 M. A. Henderson, J. Luo, A. Oliver and J. S. McIndoe, Organometallics, 2011, 30, 5471-5479.

75 X. Q. Chen, G. N. Khairallah, R. A. J. O'Hair and S. J. Williams, Tetrahedron Lett., 2011, 52, 2750-2753.

76 S. Crotti, R. Seraglia and P. Traldi, Eur. J. Mass Spectrom., 2011, 17, 85-99.

77 Z. X. Tian and S. R. Kass, J. Am. Chem. Soc., 2008, 130, 10842-10843.

78 W. T. Zhu, Y. Yuan, P. Zhou, L. Zeng, H. Wang, L. Tang, B. Guo and B. Chen, Molecules, 2012, 17, 11507-11537.

79 W. Henderson and J. S. McIndole, Mass Spectrometry of Inorganic and Organometallic Compounds, Wiley, Chichester, 2005.

80 C. Hinderling, C. Adlhart and P. Chen, Angew. Chem., Int. Ed., 1998, 37, 2685-2689.

81 C. Adlhart, C. Hinderling, H. Baumann and P. Chen, J. Am. Chem. Soc., 2000, 122, 8204-8214.

82 H. Y. Wang and J. O. Metzger, Organometallics, 2008, 27, 2761-2766.

83 H. Z. Yu, Y. Y. Jiang, Y. Fu and L. Liu, J. Am. Chem. Soc., 2010, 132, 18078-18091.

84 S. L. Zhang and Y. Q. Ding, Organometallics, 2011, 30, 633641.

85 L. M. Huffman and S. S. Stahl, J. Am. Chem. Soc., 2008, 130, 9196-9197.

86 A. Casitas, N. Ioannidis, G. Mitrikas, M. Costas and X. Ribas, Dalton Trans., 2011, 40, 8796-8799.

87 A. Casitas, A. E. King, T. Parella, M. Costas, S. S. Stahl and X. Ribas, Chem. Sci., 2010, 1, 326-330. 
88 H. J. Cristau, P. P. Cellier, J. F. Spindler and M. Taillefer, Chem.-Eur. J., 2004, 10, 5607-5622.

89 J. W. Tye, Z. Weng, A. M. Johns, C. D. Incarvito and J. F. Hartwig, J. Am. Chem. Soc., 2008, 130, 9971-9983.

90 G. O. Jones, P. Liu, K. N. Houk and S. L. Buchwald, J. Am. Chem. Soc., 2010, 132, 6205-6213.

91 C. K. Tseng, M. C. Tseng, C. C. Han and S. G. Shyu, Chem. Commun., 2011, 47, 6686-6688.

92 J. Limberger, B. C. Leal, D. F. Back, J. Dupont and A. L. Monteiro, Adv. Synth. Catal., 2012, 354, 1429-1436.

93 A. Ouali, M. Taillefer, J. F. Spindler and A. Jutand, Organometallics, 2007, 26, 65-74.

94 J. W. Tye, Z. Weng, R. Giri and J. F. Hartwig, Angew. Chem., Int. Ed., 2010, 49, 2185-2189.

95 B. M. Choudary, C. Sridhar, M. L. Kantam, G. T. Venkanna and B. Sreedhar, J. Am. Chem. Soc., 2005, 127, 9948-9949.

96 E. R. Strieter, D. G. Blackmond and S. L. Buchwald, J. Am. Chem. Soc., 2005, 127, 4120-4121.

97 A. Shafir, P. A. Lichtor and S. L. Buchwald, J. Am. Chem. Soc., 2007, 129, 3490-3491.

98 G. Franc, Q. Cacciuttolo, G. Lefevre, C. Adamo, I. Ciofini and A. Jutand, ChemCatChem, 2011, 3, 305-309.

99 L. M. Huffman and S. S. Stahl, Dalton Trans., 2011, 40, 8959-8963.

100 R. Xifra, X. Ribas, A. Llobet, A. Poater, M. Duran, M. Sola, T. D. P. Stack, J. Benet-Buchholz, B. Donnadieu, J. Mahia and T. Parella, Chem.-Eur. J., 2005, 11, 5146-5156.

101 Y. E. Corilo, F. M. Nachtigall, P. V. Abdelnur, G. Ebeling, J. Dupont and M. N. Eberlin, RSC Adv., 2011, 1, 73-78.

102 P. M. Lalli, T. S. Rodrigues, A. M. Arouca, M. N. Eberlin and B. A. D. Neto, $R S C A d v ., 2012,2,3201-3203$.
103 M. R. dos Santos, R. Coriolano, M. N. Godoi, A. L. Monteiro, H. C. B. de Oliveira, M. N. Eberlin and B. A. D. Neto, New J. Chem., 2014, 38, 2958-2963.

104 K. L. Vikse, M. A. Henderson, A. G. Oliver and J. S. McIndoe, Chem. Commun., 2010, 46, 7412-7414.

105 S. B. Duckett, C. L. Newell and R. Eisenberg, J. Am. Chem. Soc., 1994, 116, 10548-10556.

106 J. Halpern, T. Okamoto and A. Zakhariev, J. Mol. Catal., 1977, 2, 65-68.

107 P. Meakin, J. P. Jesson and C. A. Tolman, J. Am. Chem. Soc., 1972, 94, 3240-3242.

108 J. M. Brown, P. L. Evans and A. R. Lucy, J. Chem. Soc., Perkin Trans. 2, 1987, 1589-1596.

109 D. M. Chisholm, A. G. Oliver and J. S. McIndoe, Dalton Trans., 2010, 39, 364-373.

110 M. R. d. Santos, A. F. Gomes, F. C. Gozzo, P. A. Z. Suarez and B. A. D. Neto, ChemSusChem, 2012, 5, 2383-2389.

111 F. Sweet and J. D. Fissekis, J. Am. Chem. Soc., 1973, 95, 87418749.

112 L. M. Ramos, A. Y. Ponce de Leon y Tobio, M. R. dos Santos, H. C. B. de Oliveira, A. F. Gomes, F. C. Gozzo, A. L. de Oliveira and B. A. D. Neto, J. Org. Chem., 2012, 77, 1018410193.

113 I. Cepanec, M. Litvić, M. Filipan-Litvić and I. Grüngold, Tetrahedron, 2007, 63, 11822-11827.

114 M. N. Godoi, H. S. Costenaro, E. Kramer, P. S. Machado, M. G. M. D'Oca and D. Russowsky, Quim. Nova, 2005, 28, 1010-1013.

115 C. O. Kappe, J. Org. Chem., 1997, 62, 7201-7204.

116 L. M. Ramos, B. C. Guido, C. C. Nobrega, J. R. Corrêa, R. G. Silva, H. C. B. de Oliveira, A. F. Gomes, F. C. Gozzo and B. A. D. Neto, Chem.-Eur. J., 2013, 19, 4156-4168. 